\title{
O estado atual do conhecimento da diversidade dos Cladocera (Crustacea, Branchiopoda) nas águas doces do estado de Minas Gerais
}

\author{
Maria José dos Santos-Wisniewski ${ }^{1,4}$, Takako Matsumura-Tundisi ${ }^{2}$, Natália Felix Negreiros ${ }^{3}$, \\ Lidiane Cristina da Silva ${ }^{3}$, Renata Martins dos Santos ${ }^{3}$ \& Odete Rocha \\ ${ }^{1}$ Instituto de Ciências da Natureza, Universidade Federal de Alfenas - UNIFAL, \\ Rua Gabriel Monteiro da Silva, 714, Centro, CEP 37130-000, Alfenas, MG, Brasil \\ ${ }^{2}$ Instituto Internacional de Ecologia, \\ Rua Bento Carlos, 750, Centro, CEP 13560-660, São Carlos, SP, Brasil \\ ${ }^{3}$ Departamento de Ecologia e Biologia Evolutiva, Universidade Federal de São Carlos - UFSCar, \\ Rod. Washington Luis, Km 235, CP 676, CEP 13565-905, São Carlos, SP, Brasil \\ ${ }^{4}$ Autor para correspondência: Maria José dos Santos Wisniewski, e-mail: mjw@unifal-mg.edu.br
}

SANTOS-WISNIEWSKI, M.J., MATSUMURA-TUNDISI, T., NEGREIROS, N.F., SILVA, L.C., SANTOS, R.M. \& ROCHA, O. Present knowledge on Cladocera (Crustacea, Branchiopoda) diversity of freshwaters in Minas Gerais State. Biota Neotrop. 11(3): http://www.biotaneotropica.org.br/v11n3/en/abstract?inventory +bn01511032011

\begin{abstract}
The aim of this work was to present the updated checklist of Cladocera species recorded in the freshwaters of Minas Gerais state that could be used as a baseline for the ongoing or future studies in the scope of the recently initiated "Biota Minas" Program. The checklist is based on extensive literature review including recent taxonomical reviews, particularly for some genera of Chydoridae. The compiled data indicated the occurrence of 94 species of Cladocera considering 88 water bodies in Minas Gerais State. They include species from 35 genera belonging to seven among eight cladoceran families with occurrence in the freshwaters of the Neotropical region. Considering the Cladocera group as a whole, the family Chydoridae (Anomopoda Order) had the highest species richness with a total of 47 species recorded, thus corresponding to 50\% of Cladocera total richness in Minas Gerais State. Other families in decreasing order of richness were: Daphnidae with 16 species, Macrothricidae with 9, Bosminidae and Sididae with 8 species each, Moinidae and Ilyocryptidae with 3 species each. In relation to the occurrence of Cladocera in the different river basins of Minas Gerais state the data obtained encompassed registers from 6 main basins. Nevertheless, there were no records of Cladocera species for Mucuri and Paraíba do Sul river basins. It was also observed that the highest diversity of species in some river basins were probably a consequence of a higher number of studies performed at Rio Doce basin, with 67 species recorded and Rio Grande basin with 65 species. On the other hand the low number of species recorded in River Pardo basin (9 species) and River Jequitinhonha (3 species) or no record as for River Mucuri and River Paraíba do Sul basins resulted from few or none studies at all. It was concluded that there is a need to extend the coverage for other basins, particularly those located in the North and Northeast of Minas Gerais State.
\end{abstract}

Keywords: biodiversity, cladocera richness, Chydoridae, tropical cladocerans, freshwater diversity, zooplankton.

SANTOS-WISNIEWSKI, M.J., MATSUMURA-TUNDISI, T., NEGREIROS, N.F., SILVA, L.C., SANTOS, R.M. \& ROCHA, O. O estado atual do conhecimento da diversidade dos Cladocera (Crustacea, Branchiopoda) nas águas doces do estado de Minas Gerais. Biota Neotrop. 11(3): http://www.biotaneotropica.org.br/v11n3/ pt/abstract?inventory+bn01511032011

Resumo: O objetivo do presente trabalho é apresentar uma check list atualizada dos registros existentes para espécies de Cladocera nas águas doces do Estado de Minas Gerais que possa servir de base para os estudos em andamento ou estudos futuros dentro do escopo do Programa Biota Minas. O trabalho baseia-se numa extensa revisão da literatura incluindo recentes revisões taxonômicas, particularmente para alguns gêneros da família Chydoridae. O levantamento realizado evidenciou a ocorrência de 94 espécies de Cladocera, distribuídos em 88 corpos de água no estado de Minas Gerais. Estes cladóceros incluem representantes de 35 gêneros pertencentes a 7 das 8 famílias com ocorrência nas águas doces da região Neotropical. Para os Cladocera em geral, a família com maior riqueza foi a Chydoridae (Ordem Anomopoda) com um total de 47 espécies registradas, correspondendo a $50 \%$ da riqueza total dos Cladocera. As demais famílias, em ordem decrescente em relação à riqueza de espécies foram Daphnidae com 16, Macrothricidae com 9, Sididae e Bosminidae com 8 espécies cada, Moinidae e Ilyocryptidae com 3 espécies cada. Em relação à ocorrência nas bacias hidrográficas do estado de Minas Gerais, os dados obtidos evidenciaram registros de espécies de cladóceros em 6 das 8 bacias principais. Contudo não há qualquer registro para as bacias dos rios Mucuri e Paraíba do Sul. Observa-se que a maior riqueza de espécies de Cladocera em algumas bacias hidrográficas como as bacias do Rio Doce com 67 espécies, e a bacia do Rio Grande com 65 espécies resulta muito provavelmente do maior número de estudos realizados nas mesmas, e que por outro lado, bacias com baixa riqueza de Cladocera como as bacias do rio Pardo ( 9 espécies) e do Rio 
Jequitinhonha (3 espécies) ou com nenhuma ocorrência registrada (bacias do rio Mucuri e do rio Paraíba do Sul) resultam de poucos, ou nenhum estudo, respectivamente. Conclui-se que há necessidade de ampliação da cobertura geográfica para amostragem de corpos de água em outras bacias, particularmente aquelas localizadas na região norte e nordeste do estado de Minas Gerais.

Palavras-chave: biodiversidade, riqueza de cladocera, Chydoridae, cladóceros tropicais, diversidade em água doce, zooplâncton.

\section{Introdução}

O conhecimento da biodiversidade brasileira é ainda bastante incompleto para a maioria dos grupos de seres vivos. Este fato constitui um entrave para o desenvolvimento de ações de preservação e ou de conservação da diversidade biológica, uma condição imprescindível para o desenvolvimento sustentável. É também amplamente reconhecido que atualmente a perda de biodiversidade ocorre de forma acelerada fazendo com que muitas espécies não sejam provavelmente identificadas antes de serem extintas.

Contudo, na última década muitos esforços tem sido empreendidos por parte dos governos e das agências de fomento visando diminuir as lacunas no conhecimento da biodiversidade em âmbito nacional por meio de programas como o Programa Nacional de Biodiversidade do Ministério do Meio Ambiente e o Programa o Biota Fapesp para o Estado de São Paulo, que já contam com mais de uma década (Ismael et al. 1999) e o Biota Minas recentemente implementado (Maia Barbosa et al. 2009). As águas doces constituem atualmente um dos ecossistemas com maior ameaça de perda de biodiversidade, pela elevada vulnerabilidade desses sistemas devido a uma conjunção de fatores como: poluição generalizada, eutrofização, invasão por espécies exóticas, alteração e ou destruição dos habitats (Tundisi 2003, Rocha et al. 2005).

Em águas doces a comunidade zooplanctônica é constituída por representantes de diversos grupos de invertebrados, entre os quais os Cladocera que desempenham um papel de grande relevância por atuarem como elo na transferência de energia dos produtores primários (algas) para os níveis tróficos superiores.

Uma das dificuldades para o desenvolvimento de ações voltadas para a proteção da diversidade biológica no Brasil é o fato do conhecimento além de ser restrito se encontrar disperso em veículos de difícil acesso. Há ainda grande carência de trabalhos de síntese que não só reúnam as informações existentes, mas também analisem os padrões de distribuição geográfica das espécies, sua origem e os prováveis fatores determinantes.

O presente trabalho visa sintetizar e atualizar o conhecimento sobre a diversidade dos Cladocera nas águas doces do Estado de Minas Gerais por meio de uma extensa revisão da literatura incluindo trabalhos recentes de revisão taxonômica, particularmente de alguns gêneros da família Chydoridae (Smirnov 1998, Hudec 2000, van Damme et al. 2003, Kotov 2003, Kotov \& Sinev 2004, Sinev \& Hollwedel 2005, Sinev et al. 2005, van Damme \& Dumont 2008, Kotov 2009, van Damme et al. 2010, Sinev \& Elmoor-Loureiro 2010). Este último tendo resultado na descrição de 3 novas espécies para o Brasil.

\section{Materiais e Métodos}

Esta síntese foi produzida com base em uma ampla revisão de trabalhos publicados em periódicos e livros, e dados não publicados (teses, dissertações, monografias, relatórios de projetos e relatórios de iniciação científica) além de informações disponíveis em sites da internet. Todos os registros foram considerados, mesmo existindo dúvidas quanto à real ocorrência da espécie na região Neotropical. Os nomes das espécies registradas foram mantidos tal como nos registros originais feitos pelos autores dos estudos, explicitando-se, no entanto, os atuais nomes válidos em face às revisões taxonômicas realizadas nos últimos 15 anos.

A classificação das bacias hidrográficas seguiu o critério do Instituto Mineiro de Gestão das Águas (IGAM), o qual divide o estado de Minas Gerais em nove bacias: Bacia do Rio São Francisco, Bacia do Rio Grande, Bacia do rio Paranaíba, Bacia do Rio Doce, Bacia do Jequitinhonha, Bacia do Rio Pardo, Bacia do Rio Paraíba do Sul, bacia do Rio Piracicaba e Jaguari, Bacia do Leste e Bacia do Rio Mucuri.

\section{Resultados}

A relação das espécies de Cladocera com ocorrência registrada no estado de Minas Gerais é apresentada na Tabela 1, juntamente com números correspondentes aos corpos de água onde foram encontradas. Na Tabela 2 são relacionados os corpos de água enumerados com a mesma numeração atribuída na Tabela 1 , as respectivas coordenadas geográficas e os autores dos estudos neles realizados.

A compilação dos registros das espécies resultou em um total de 94 espécies de Cladocera, distribuídos em 88 corpos de água no estado de Minas Gerais. Estes cladóceros incluem representantes de 35 gêneros pertencentes a 7 das 8 famílias com ocorrência nas águas doces da região Neotropical (Tabela 1).

Na Ordem Ctenopoda só ocorreram representantes da família Sididae, com um total de 8 espécies, distribuídas em 3 gêneros. Entre estes, se destacou o gênero Diaphanosoma, com a ocorrência de 6 espécies.

Para a Ordem Anomopoda a maior riqueza de espécies foi encontrada para a família Chydoridae. Esta é a família com maior riqueza de táxons nas águas doces de Minas Gerais, com 47 espécies registradas até o presente, correspondendo a $50 \%$ do total de espécies com ocorrência neste estado. As demais famílias, em ordem decrescente em relação à riqueza de espécies, foram Daphnidae com 16 espécies, Macrothricidae com 9, Bosminidae com 8, Moinidae e Ilyocryptidae, com 3 espécies cada.

Em relação à ocorrência nas bacias hidrográficas do estado de Minas Gerais, os dados obtidos evidenciaram registros de cladóceros em 6 das 8 bacias principais. Nenhum registro foi obtido para as bacias dos rios Mucuri e Paraíba do Sul (Figura 1). Representantes das famílias Bosminidae e Daphnidae foram registrados em 6 bacias.

Em relação à contribuição relativa das famílias para a riqueza de espécies das associações de Cladocera, as famílias Chydoridae e Daphnidae foram as mais representativas, contribuindo com cerca de $50 \%$ ou mais do total de espécies (Figura 1) em cada bacia nas quais tiveram ocorrência registrada. Os Chydoridae representaram $51 \%$ e $49 \%$ do total de espécies nas bacias do Rio Grande e do Rio Doce, e os Bosminidae foram os mais representativos na bacia dos rios Jequitinhonha $(67 \%)$ e Pardo (45\%). Por outro lado as famílias Ilyocryptidae e Moinidae foram as menos representativas em termos de riqueza de espécies em várias bacias hidrográficas (Figura 1).

A análise comparativa da riqueza de espécies de Cladocera em geral entre as bacias hidrográficas evidenciou que as bacias com maior riqueza de espécies foram as bacias do Rio Doce, com a ocorrência de 67 espécies, e a Bacia do Rio Grande, com 65 espécies. Por outro lado os menores valores de riqueza de espécies foram obtidos para 
Tabela 1. Classificação taxonômica (tal como constam nos trabalhos originais) e ocorrência das espécies de Cladocera registradas no estado de Minas Gerais. Os números indicam as localidades onde as espécies foram registradas (ver Tabela 2), e entre parênteses encontra-se a nomenclatura atualmente utilizada para as espécies.

Table 1. Taxonomic classification (as listed in the original works) and occurrence of species of Cladocera recorded in Minas Gerais state. The numbers indicate the locations where species were recorded (see Table 2), and in parentheses is the nomenclature currently used for the species.

Classificação taxonômica

\section{Família Bosminidae}

Bosmina coregoni Baird, 1857 (identificação duvidosa)

Bosmina eurynotus (identificação duvidosa)

Bosmina freyi De Melo and Hebert, 1994

Bosmina hagmanni Stingelin, 1904

Bosmina longirostris (O.F. Muller, 1785)

Bosmina longiseta (identificação duvidosa)

Bosmina tubicen Brehm, 1953

Bosminopsis deitersi Richard, 1895

\section{Família Chydoridae}

Acroperus harpae Baird, 1835 (Acroperus tupinamba Sinev \& Elmoor-Loureiro, 2010)

Acroperus tupinamba Sinev \& Elmoor-Loureiro, 2010

Alona affinis Leydig, 1860 (Alona ossiani Sinev, 1998)

Alona cambouei Guerne \& Richard, 1893 (Alona glabra Sars, 1901)

Alona costata Sars, 1862 (Alona iheringula Sars, 1901)

Alona excisa (identificação duvidosa, provavelmente Alonella excisa)

Alona dentifera (Sars, 1901)

Alona glabra Sars, 1901

Alona guttata Sars, 1862

Alona intermedia Sars, 1862

Alona monacantha (Coronatella monacantha (Sars, 1901)

Alona ossiani Sinev, 1998

Alona poppei (Coronatella poppei (Richard, 1897)

Alona rectangula (Coronatella rectangula (Sars, 1861)

Alona setigera Brehm, 1931

Alona quadrata (identificação duvidosa)

Alona quadrangularis (O.F. Muller, 1875) (Alona yara Sinev \& Elmoor-Loureiro, 2010)

Alona verrucosa Sars, 1901

Alona yara Sinev \& Elmoor-Loureiro, 2010

Alonella chlathratula Sars, 1896

Alonella dentifera (Alona dentifera (Sars, 1901)

Alonella dadayi Birge, 1910

Alonella karua (Karualona muelleri (Richard, 1897)

Alonella lineolata Sars, 1901

Alonella nana (Baird, 1850) (identificação duvidosa)

Biapertura affinis (Alona ossiani Sinev, 1998)

Biapertura intermedia (Alona intermedia Sars, 1862)

Biapertura karua (Karualona muelleri (Richard, 1897)
Localização

$$
\begin{gathered}
1,2,4,7,88 \\
1 \\
51,75 \\
1,2,3,5,6,10,12,16,17,20,24,29,31,40, \\
47,49,51,52,55,58,64,68,69,70,71,72, \\
73,75,77,78,79,80,81,84,85,88 \\
35,47,51,52,53,54,55,65,66,69,71,73,75,77,78,79,8 \\
0,81,83,84,85,86,87,88 \\
79 \\
1,2,3,5,6,9,10,11,12,13,14,15,16,17,18,20,29,31,42 \\
, 44,45,49,50,51,56,64,68,74,75,83,84,85,86 \\
1,2,4,5,9,10,11,12,13,14,17,19,20,23,24,25,28,29,3 \\
0,31,35,36,37,38,39,40,43,48,49,50,51,53,56,59,60 \\
, 64,66,67,68,69,70,71,72,73,74,75,77,78,79,80,81, \\
82,83,84,85,87,88
\end{gathered}
$$

$32,33,38,59,60,67,72,75$

$$
\begin{gathered}
75 \\
8 \\
1,5 \\
22,28,30,41 \\
32,38 \\
5,68 \\
75
\end{gathered}
$$

$1,2,5,21,22,28,29,30,31,32,38,41,45,51,56,59,60$, 72,75 $28,59,60,67,75$

5,32

67,75

$1,5,31,33$

$1,2,8,28,29,31,38,44,59,75,76,82$

75

$2,3,5,12,16$

44

$2,72,75$

75

1,75

5

$8,22,67,75$

8

1

2

$2,3,5,12,16$

$5,27,29,31,32,33$

5,75 
Tabela 1. Continuação...

Classificação taxonômica

Biapertura verrucosa (Alona verrucosa Sars, 1901)

Camptocercus australis Sars, 1896

Camptocercus dadayi Stingelin, 1913 (Camptocercus australis Sars, 1896)

Coronatella monacantha Sars, 1901

Coronatella poppei Richard, 1897

Chydorus dentifer Daday, 1905

Chydorus eurynotus Sars, 1901

Chydorus globosus (Pseudochydorus globosus, Baird, 1850)

Chydorus hybridus (Ephemeroporus hybridus (Daday, 1905)

Chydorus parvireticulatus Frey, 1987

Chydorus pubescens Sars, 1901

Chydorus sphaericus sens. lat.

Dadaya macrops (Daday, 1898)

Disparalona dadayi (Alonella dadayi Birge, 1910)

Dunhevedia odontoplax Sars, 1901

Ephemeroporus barroisi (Richard, 1894)

Ephemeroporus hybridus (Daday, 1905)

Ephemeroporus tridentatus (Bergamin, 1931)

Euryalona brasiliensis Brehm \& Thomsen, 1936

Euryalona orientalis (Daday, 1898)

Graptoleberis testudinaria (Fischer, 1848)

(Graptoleberis occidentalis, Sars, 1901)

Kurzia latissima (Daday, 1898) (Kurzia polyspina Hudec, 2000)

Kurzia polyspina Hudec, 2000

Leydigia ciliata Gauthier, 1939 (Leydigia striata, Berabén, 1939)

Leydigia ipojucae Brehm, 1938

Leydigia propinqua Sars, 1903

Leydigia striata Berabén, 1939

Leydigiopsis curvirostris Sars, 1901

Leydigiopsis ornata Daday, 1905

Nicsmirnovius eximius Kiser, 1948)

Notoalona sculpta (Sars, 1901)

Oxyurella ciliata Bergamin, 1931

Parvalona parva (Daday, 1905)

Pleuroxus scopuliferus (Picripleuroxus similis (Vávra, 1900)

Pleuroxus similis (Picripleuroxus similis (Vávra, 1900)

\section{Família Daphnidae}

Ceriodaphnia cornuta Sars, 1886

Ceriodaphnia cornuta cornuta

Ceriodaphnia cornuta intermedia

Ceriodaphnia cornuta rigaudi

Ceriodaphnia dubia Richard, 1894 (identificação duvidosa)

Ceriodaphnia reticulata (Jurine, 1820)

Ceriodaphnia richardi Sars, 1901

Ceriodaphnia rigaudi (Ceriodaphnia cornuta Sars, 1886)

Ceriodaphnia silvestrii Daday, 1902

Ceriodaphnia quadrangula (O.F. Müller, 1785)
Localização

1

75

67,75

75

75

75

$1,2,5,67,75$

$4,7,8,34$

7

75

$2,3,5,8,12,16,75$

$1,2,5,12,16,67,75$

1,5

$1,38,50,75$

1

$1,8,20,21$

67,75

1,75

75

$72,75,81,83,85$

38,75

75

75

48,51

29

75

75

$1,67,69,75$

1

75

1

1,75

75

75

67

$1,2,5,6,7,9,10,14,17,18,19,22,24,25,27,29,30,46,47$ ,51,52,53,54,55,57,58,60,61,62,66,68,69,70,71,72, $73,74,75,76,77,78,80,81,82,84,87,88$

$1,2,21,51,75$

$50,51,75$

$1,2,47,51,52,55,75,77,81$

7,88

80

$24,57,70,71,73,78,80$

84,85

$1,2,5,12,16,51,69,75$

71

$31,61,62,65,75,83,84$ 
Tabela 1. Continuação...

\begin{tabular}{|c|c|}
\hline Classificação taxonômica & Localização \\
\hline Daphnia dubia (identificação duvidosa) & 68 \\
\hline Daphnia galeata (identificação duvidosa) & $68,76,88$ \\
\hline Daphnia gessneri Herbst, 1967 & $\begin{array}{c}1,2,5,6,9,12,24,25,30,51,52,53,55,57,68,69,70,71,7 \\
2,73,74,75,77,78,79,80,81,88\end{array}$ \\
\hline Daphnia laevis Birge, 1878 & $1,5,6,12,20,51,52,55,57,64,68,75$ \\
\hline Scapholeberis armata (Herrick, 1882) & 1 \\
\hline Scapholeberis kingi Sars, 1903 & 7 \\
\hline $\begin{array}{l}\text { Scapholeberis mucronata (O.F. Müller, 1785) } \\
\text { (Scapholeberis armata (Herrick, 1882)) }\end{array}$ & 80 \\
\hline Simocephalus latirostris Stingelin, 1906 & 75 \\
\hline Simocephalus serrulatus Koch, 1841 & $1,2,3,8,12,16,31,61,62,69,73,75,80$ \\
\hline Simocephalus vetulus (O.F. Müller, 1776) & $1,68,75$ \\
\hline \multicolumn{2}{|l|}{ Família Ilyocryptidae } \\
\hline Ilyocryptus sordidus (Liévin, 1848) & 23,38 \\
\hline Ilyocryptus spinifer Herrick, 1882 & $1,2,4,5,7,8,29,31,33,37,39,40,41,51,69,72,75,77,81$ \\
\hline Ilyocryptus verrucosus Daday, 1905 & 69,75 \\
\hline \multicolumn{2}{|l|}{ Família Macrothricidae } \\
\hline Grimaldina brazzai Richard, 1892 & $5,41,65,72$ \\
\hline Macrothrix elegans Sars, 1901 & 5,75 \\
\hline Macrothrix flabelligera Smirnov, 1992 & $1,75,81$ \\
\hline Macrothrix laticornis (Jurine, 1820) & $2,3,5,7,8,12,16,21,22,29,41$ \\
\hline Macrothrix paulensis (Sars, 1901) & 1,75 \\
\hline Macrothrix spinosa King, 1853 & $1,69,75$ \\
\hline Macrothrix superaculeata (Smirnov, 1992) & 72,75 \\
\hline Macrothrix triserialis (Brady, 1886) & 67 \\
\hline Streblocerus pygmaeus Sars, 1901 & 41 \\
\hline \multicolumn{2}{|l|}{ Família Moinidae } \\
\hline Moina micrura Kurz, 1874 & $30,31,42,44,45,52,54,55,61,62,75,78,80,81$ \\
\hline Moina minuta Hansen, 1899 & $\begin{array}{c}1,5,9,11,24,25,29,30,31,42,43,47,49,50,51,52,53 \\
57,63,66,68,69,70,71,72,73,74,75,77,78,79,80,81 \\
84,85,88\end{array}$ \\
\hline Moina reticulata (Daday, 1905) & $5,7,8$ \\
\hline \multicolumn{2}{|l|}{ Familia Sididae } \\
\hline Diaphanosoma birgei Korineck, 1981 & $\begin{array}{c}1,2,3,5,10,12,13,14,15,16,20,30,38,43,47,51,52,55 \\
61,62,66,68,69,70,72,75,81,83,84,85\end{array}$ \\
\hline $\begin{array}{l}\text { Diaphanosoma brachyurum (Lievin, 1848) (Diaphanosoma birgei Korinek, } \\
\text { 1981) }\end{array}$ & $1,4,5,7,8,53$ \\
\hline Diaphanosoma brevireme Sars, 1901 & $1,9,11,69,72,75,81$ \\
\hline Diaphanosoma fluviatile Hansen, 1899 & $16,49,51,72,75,81$ \\
\hline Diaphanosoma polyspina Korovchinsky 1982 & 69 \\
\hline Diaphanosoma spinulosum Herbst, 1967 & $1,30,51,68,69,70,72,75,81$ \\
\hline Latonopsis australis Sars, 1888 & 77 \\
\hline Pseudosida bidentata Herrick, 1884 & 1,5 \\
\hline Pseudosida ramosa (Daday, 1904) & 5 \\
\hline
\end{tabular}


Tabela 2. Lista dos corpos de água do estado de Minas Gerais nos quais foram registradas as espécies de Cladocera relacionadas na Tabela 1, coordenadas geográficas e autores dos estudos. Entre parênteses constam os números correspondentes aos corpos d'água apresentados na Tabela 1.

Table 2. List of water bodies of Minas Gerais state where the Cladocera species listed in Table 1 were registered, geographical coordinates and study authors. In brackets are the numbers corresponding to the water bodies shown in Table 1.

\begin{tabular}{ccc}
\hline Bacia & Corpos d'água & Coordenadas geográficas \\
\hline Rio Doce & Lago Dom Helvécio (1) & $42^{\circ} 35^{\prime}-42^{\circ} 40^{\prime} \mathrm{W}$ anda \\
& & $19^{\circ} 45^{\prime}-19^{\circ} 50^{\prime} \mathrm{S}$
\end{tabular}

Lagoa Carioca (2)

Lagoa Aguapé (3)

Lagoa Bonita (4)

Lagoa Amarela (5)

Lagoa Jacaré (6)

Lagoa Belgo-Mineira (7)

Lagoa 33 (8)

Lagoa Palmeiras (9)

Lagoa Almecega (10)

Lagoa Carvão com Azeite (11)

Lagoa Águas Claras (12)

Lagoa Recreio (13)

Lagoa Poço Fundo (14)

Lagoa Verde (15)

Lagoa Ariranha (16)

Lagoa Palmeirinha (17)

Lagoa Anibal (18)

Lagoa Ferrugem (19)

Lago Gambazinho (20)

Lago Hortência (21)

Lago Pedra (22)

Reservatório de Dona Rita (23)

Reservatório Peti (24)

Reservatório Salto Grande (25) $42^{\circ} 37^{\prime} 12^{\prime \prime} \mathrm{W}$ and $19^{\circ} 45^{\prime} 20^{\prime \prime} \mathrm{S}$
$42^{\circ} 38^{\prime} 39^{\prime \prime} \mathrm{W}$ and $19^{\circ} 51^{\prime} 00^{\prime \prime} \mathrm{S}$ $42^{\circ} 35^{\prime}-42^{\circ} 40^{\prime} \mathrm{W}$ and $19^{\circ} 45^{\prime}-19^{\circ} 50^{\prime \prime} \mathrm{S}$ $42^{\circ} 34^{\prime} 35^{\prime \prime} \mathrm{W}$ and $19^{\circ} 49^{\prime} 16^{\prime \prime} \mathrm{S}$ $42^{\circ} 35^{\prime}-42^{\circ} 40^{\prime} \mathrm{W}$ and $19^{\circ} 45^{\prime}-19^{\circ} 50^{\prime \prime} \mathrm{S}$

$42^{\circ} 35^{\prime}-42^{\circ} 40^{\prime} \mathrm{W}$ and $19^{\circ} 45^{\prime}-19^{\circ} 50^{\prime \prime} \mathrm{S}$ $42^{\circ} 35^{\prime}-42^{\circ} 40^{\prime} \mathrm{W}$ and $19^{\circ} 45^{\prime}-19^{\circ} 50^{\prime \prime} \mathrm{S}$

$42^{\circ} 35^{\prime} 47^{\prime \prime} \mathrm{W}$ and $19^{\circ} 49^{\prime} 02$ " S

$42^{\circ} 34^{\prime} 05^{\prime \prime} \mathrm{W}$ and $19^{\circ} 49^{\prime} 07^{\prime \prime} \mathrm{S}$
Brito et al. 2002, Brandão et al. 2002, Bezerra-Neto \& Pinto-Coelho 2008, Dumont \& Tundisi 1997, Eskinazi-Sant' Anna et al. 2005, Maia-Barbosa et al. 2008, Matsumura-Tundisi 1997, Matsumura-Tundisi et al. 1984, Miranda 2005, Pinto-Coelho et al. 2008, Rocha \& Matsumura-Tundisi 1997, Santos 1980, Tundisi et al. 1997

Brandão et al. 2002, Dumont \& Tundisi 1997, Eskinazi-Sant'Anna et al. 2005,

Matsumura-Tundisi et al. 1997,

Moretto 2001, Pinto-Coelho et al. 2008, Santos 1980, Tundisi et al. 1997

Moretto 2001

Santos 1980

Eskinazi-Sant'Anna et al. 2005, Moretto 2001, Pinto-Coelho et al. 2008, Tundisi et al. 1997

Dumont \& Tundisi 1997,

Eskinazi-Sant'Anna et al. 2005,

Pinto-Coelho et al. 2008, Tundisi et al. 1997 Santos 1980

Dumont \& Tundisi 1997, Santos 1980

Tundisi et al. 1997

Tundisi et al. 1997

Tundisi et al. 1997

Brito et al. 2002,

Eskinazi-Sant'Anna et al. 2005, Moretto 2001, Tundisi et al. 1997

Tundisi et al. 1997

Tundisi et al. 1997

Tundisi et al. 1997

Moretto 2001, Tundisi et al. 1997

Eskinazi-Sant'Anna et al. 2005, Pinto-Coelho et al. 2008,

Tundisi et al. 1997

Tundisi et al. 1997b

Tundisi et al. 1997

Brandão et al. 2002,

Eskinazi-Sant'Anna et al. 2005, Pinto-Coelho et al. 2008,

Eskinazi-Sant'Anna et al. 2005, López 1996

López et al. 1996

Eskinazi-Sant'Anna et al. 2005

Eskinazi-Sant'Anna et al. 2005

Eskinazi-Sant'Anna et al. 2005 
Tabela 2. Continuação...

\begin{tabular}{|c|c|c|c|}
\hline Bacia & Corpos d'água & Coordenadas geográficas & Autores \\
\hline & Rio Barão dos Coquais (26) & & Eskinazi-Sant'Anna et al. 2005 \\
\hline & Rio Caraçá (27) & & $\begin{array}{l}\text { Eskinazi-Sant'Anna et al. 2005, } \\
\text { Menendez \& Maia-Barbosa } 2002\end{array}$ \\
\hline & Rio Congonhas (28) & & Eskinazi-Sant' Anna et al. 2005 \\
\hline & Rio Doce (29) & & $\begin{array}{l}\text { Eskinazi-Sant'Anna et al. 2005, } \\
\text { Menendez \& Maia-Barbosa } 2002\end{array}$ \\
\hline & Rio Piracicaba (30) & $42^{\circ} 47^{\prime} \mathrm{W} / 19^{\circ} 30^{\prime} \mathrm{S}$ & $\begin{array}{l}\text { Eskinazi-Sant'Anna et al. 2005, } \\
\text { Menendez \& Maia-Barbosa } 2002\end{array}$ \\
\hline & Rio Santa Bárbara (31) & $42^{\circ} 47^{\prime} 274^{\prime \prime} \mathrm{W} / 19^{\circ} 30^{\prime} 828^{\prime \prime} \mathrm{S}$ & Menendez \& Maia-Barbosa 2002 \\
\hline & Rio Severo (32) & & $\begin{array}{l}\text { Eskinazi-Sant'Anna et al. 2005, } \\
\text { Menendez \& Maia-Barbosa } 2002\end{array}$ \\
\hline & Rio Peixe (33) & & Menendez \& Maia-Barbosa 2002 \\
\hline & Rio Ipanema (34) & & Eskinazi-Sant'Anna et al. 2005 \\
\hline & $\begin{array}{l}\text { Parque Estadual do Itacolomi, OP } \\
\text { (35) }\end{array}$ & & Eskinazi-Sant'Anna et al. 2005 \\
\hline & Lagoa Teobaldo (36) & $43^{\circ} 27^{\prime} 57^{\prime \prime} \mathrm{W} / 20^{\circ} 25^{\prime} 55^{\prime \prime} \mathrm{S}$ & Freitas \& Eskinazi-Sant'Anna 2009 \\
\hline \multirow[t]{23}{*}{ São Francisco } & Lagoa Azul (37) & $45^{\circ} 40^{\prime} \mathrm{W} / 18^{\circ} 43^{\prime} \mathrm{S}$ & $\begin{array}{c}\text { Dabés 1995, } \\
\text { Eskinazi-Sant'Anna et al. } 2005\end{array}$ \\
\hline & Lagoa Cipó (38) & $44^{\circ} 55^{\prime} \mathrm{W} / 17^{\circ} 43^{\prime} \mathrm{S}$ & $\begin{array}{c}\text { Dabés 1995, } \\
\text { Eskinazi-Sant'Anna et al. } 2005\end{array}$ \\
\hline & Lagoa Praiano (39) & $44^{\circ} 55^{\prime} \mathrm{W} / 17^{\circ} 43^{\prime} \mathrm{S}$ & $\begin{array}{c}\text { Dabés 1995, } \\
\text { Eskinazi-Sant'Anna et al. } 2005\end{array}$ \\
\hline & Lagoa Tacho (40) & $44^{\circ} 55^{\prime} \mathrm{W} / 17^{\circ} 43^{\prime} \mathrm{S}$ & $\begin{array}{c}\text { Dabés 1995, } \\
\text { Eskinazi-Sant'Anna et al. } 2005\end{array}$ \\
\hline & Lagoa Comprida (41) & & Eskinazi-Sant'Anna et al. 2005 \\
\hline & Lago de Confins (42) & & Eskinazi-Sant'Anna et al. 2005 \\
\hline & Lago Feia (43) & & $\begin{array}{l}\text { Eskinazi-Sant'Anna et al. } 2005, \\
\text { Maia-Barbosa et al. } 2003\end{array}$ \\
\hline & Lago Mares (44) & & Eskinazi-Sant'Anna et al. 2005 \\
\hline & Lagoa Santa (45) & & Eskinazi-Sant'Anna et al. 2005 \\
\hline & Lago Sumidouro (46) & & Eskinazi-Sant'Anna et al. 2005 \\
\hline & Rio Itapecerica (47) & $44^{\circ} 53^{\prime} 17^{\prime \prime} \mathrm{W} / 20^{\circ} 08^{\prime} 21^{\prime \prime} \mathrm{S}$ & Ferraz et al. 2009 \\
\hline & Represa Estaleiro (48) & $45^{\circ} 20^{\prime} 33^{\prime \prime} \mathrm{W} / 18^{\circ} 36^{\prime} 10^{\prime \prime} \mathrm{S}$ & Sampaio 2002 \\
\hline & Represa Fazenda BMG (49) & $45^{\circ} 21^{\prime} 19^{\prime \prime} \mathrm{W} / 18^{\circ} 36^{\prime} 48^{\prime \prime} \mathrm{S}$ & Sampaio 2002 \\
\hline & Represa Parque de Exposições (50) & $45^{\circ} 21^{\prime} 44^{\prime \prime} \mathrm{W} / 18^{\circ} 36^{\prime} 07^{\prime \prime} \mathrm{S}$ & Sampaio 2002 \\
\hline & Reservatório de Três Marias (51) & $45^{\circ} 15^{\prime} 44^{\prime \prime} \mathrm{W} / 18^{\circ} 13^{\prime} 25^{\prime \prime} \mathrm{S}$ & Brito 2010, Sampaio 2002 \\
\hline & Reservatório da Pampulha (52) & $43^{\circ} 56^{\prime} 47^{\prime \prime} \mathrm{W} / 19^{\circ} 55^{\prime} 09^{\prime \prime} \mathrm{S}$ & $\begin{array}{c}\text { Bezerra-Neto \& Pinto-Coelho 2003, } \\
\text { Costa 1998, Eskinazi-Sant'Anna et al. } \\
\text { 2005, Macedo \& Pinto-Coelho 1997, } \\
\text { 2000a,b,c, Pinto-Coelho 1998, } \\
\text { Pinto-Coelho 2010, Pinto-Coelho \& } \\
\text { Greco 1998, 1999, Pinto-Coelho et al. } \\
\text { 1997a,b, 2003, 2005c, }\end{array}$ \\
\hline & Reservatório Vargem das Flores (53) & $44^{\circ} 10^{\prime} \mathrm{W} / 19^{\circ} 55^{\prime} \mathrm{S}$ & $\begin{array}{c}\text { Freire \& Pinto-Coelho 1986, } \\
\text { Jaramillo-Londoño \& } \\
\text { Pinto-Coelho } 2010\end{array}$ \\
\hline & Lagoa do Nado (54) & $43^{\circ} 57^{\prime} 34^{\prime \prime} \mathrm{W} / 19^{\circ} 49^{\prime} 56^{\prime \prime} \mathrm{S}$ & $\begin{array}{l}\text { Bezerra-Neto 2001, } \\
\text { Bezerra-Neto \& Pinto-Coelho 2002, } \\
\text { Eskinazi-Sant'Anna et al. } 2005\end{array}$ \\
\hline & Reservatório de Ibirité (55) & & Pinto-Coelho et al. 1998 \\
\hline & $\begin{array}{l}\text { Lagoa Ólhos d' água - em Lagoa } \\
\text { Santa (56) }\end{array}$ & & $\begin{array}{c}\text { Eskinazi-Sant'Anna et al. 2005, } \\
\text { Landa \& Torres } 2004\end{array}$ \\
\hline & Reservatório de Cajuru (57) & & Eskinazi-Sant'Anna et al. 2005 \\
\hline & Reservatório de Gafanhoto (58) & & Eskinazi-Sant'Anna et al. 2005 \\
\hline & Rio Indaiá (59) & & Eskinazi-Sant'Anna et al. 2005 \\
\hline
\end{tabular}


Tabela 2. Continuação...

\begin{tabular}{|c|c|c|c|}
\hline Bacia & Corpos d'água & Coordenadas geográficas & Autores \\
\hline & Rio Preto do Itambé (60) & & Eskinazi-Sant'Anna et al. 2005 \\
\hline & $\begin{array}{l}\text { Represa da Reitoria e Quatro } \\
\text { Pilastras, UFV (61) }\end{array}$ & $42^{\circ} 52^{\prime} \mathrm{W} / 20^{\circ} 45^{\prime}$ & Peixoto \& Okano 2004 \\
\hline & $\begin{array}{l}\text { Represa de Quatro Pilastras, UFV } \\
\text { (62) }\end{array}$ & $42^{\circ} 52^{\prime} \mathrm{W} / 20^{\circ} 45^{\prime}$ & Peixoto \& Okano 2004 \\
\hline & $\begin{array}{l}\text { Tratamento de efluentes sanitários e } \\
\text { de piscicultura - Viçosa (63) }\end{array}$ & $42^{\circ} 52^{\prime} \mathrm{W} / 20^{\circ} 45^{\prime}$ & Souza 2007 \\
\hline & $\begin{array}{l}\text { Lago Massacará - Bacia São } \\
\text { Francisco (64) }\end{array}$ & $44^{\circ} 34^{\prime} \mathrm{W} / 19^{\circ} 27^{\prime} \mathrm{S}$ & Sampaio \& López 2000 \\
\hline \multirow[t]{10}{*}{ Paranaíba } & Rio Uberabinha (65) & & Cardoso et al. 2008 \\
\hline & Lago do Parque Sabiá (66) & $48^{\circ} 14^{\prime} 02^{\prime \prime} \mathrm{W} / 18^{\circ} 54^{\prime} 52^{\prime \prime} \mathrm{S}$ & Alemeida et al. 2007 \\
\hline & $\begin{array}{l}\text { Lagoas de vereda do Clube Caça } \\
\text { e Pesca Itororó de Uberlândia (67) }\end{array}$ & $48^{\circ} 18^{\prime} \mathrm{W} / 18^{\circ} 59^{\prime \prime} \mathrm{S}$ & Pinese 2008 \\
\hline & Reservatório de Emborcação (68) & $47^{\circ} 59^{\prime} 34,6 ” \mathrm{~W} / 18^{\circ} 31^{\prime} 10,3^{\prime \prime} \mathrm{S}$ & $\begin{array}{c}\text { Eskinazi-Sant'Anna et al. 2005, } \\
\text { Ramos et al. } 2008\end{array}$ \\
\hline & $\begin{array}{l}\text { Reservatório Marechal Mascarenhas } \\
\text { de Moraes (69) }\end{array}$ & & Rosa 2008 \\
\hline & Rio Araguari (70) & & Gomes e Souza \& von Sperling 2005 \\
\hline & Reservatório de Miranda (71) & & Eskinazi-Sant'Anna et al. 2005 \\
\hline & Cachoeira Dourada (72) & & Peret 2007 \\
\hline & Reservatório Nova Ponte (73) & & Eskinazi-Sant'Anna et al. 2005 \\
\hline & Reservatório São Simão (74) & & Eskinazi-Sant'Anna et al. 2005 \\
\hline \multirow[t]{12}{*}{ Rio Grande } & Reservatório de Furnas (75) & & $\begin{array}{l}\text { Brito 2010, Castilho et al. 2009, } \\
\text { Corgosinho \& Pinto-Coelho 2006, } \\
\text { Del Águila 2001, Eskinazi-Sant' Anna } \\
\text { et al. 2005, Ferrari 2007, Franco et al } \\
\text { 2005, Leone 2009, Pinto-Coelho et al. } \\
\text { 1997b, Paes et al. 2008, Pinto-Coelho } \\
\text { \& Corgosinho 1998, Rocha 2006, Rosa } \\
\text { 2008, Santeiro \& Pinto-Coelho 2000, } \\
\text { Santeiro et al. 2006, Santos 2007, é } \\
\text { Santos et al. 2007, 2008, 2009, Santos- } \\
\text { Wisniewski et al. 2005, } \\
\text { Santos-Wisniewski 2009, Silva 2011, } \\
\text { Silva et al. 2007, Silva et al. } 2009, \\
\text { Sipaúba-Tavares et al. } 2009\end{array}$ \\
\hline & Lago Marimbondo (76) & & Eskinazi-Sant'Anna et al. 2005 \\
\hline & Reservatório de Camargo (77) & & Eskinazi-Sant' Anna et al. 2005 \\
\hline & Reservatório de Igarapava (78) & & Eskinazi-Sant' Anna et al. 2005 \\
\hline & Reservatório de Itutinga (79) & & Eskinazi-Sant'Anna et al. 2005 \\
\hline & Reservatório de Jaguara (80) & & Eskinazi-Sant'Anna et al. 2005 \\
\hline & Reservatório Volta Grande (81) & & Eskinazi-Sant'Anna et al. 2005 \\
\hline & Rio São Gonçalo (82) & & Eskinazi-Sant'Anna et al. 2005 \\
\hline & Represa Nova - UFLA (83) & $45^{\circ} 00^{\prime} 10^{\prime \prime} \mathrm{W} / 21^{\circ} 14^{\prime} 30^{\prime \prime} \mathrm{S}$ & Landa \& Mourgués-Schurter 2000a \\
\hline & Represa Estação - UFLA (84) & $45^{\circ} 00^{\prime} 10^{\prime \prime} \mathrm{W} / 21^{\circ} 14^{\prime} 30^{\prime \prime} \mathrm{S}$ & Landa \& Mourgués-Schurter 2000a \\
\hline & Represa Zootecnia - UFLA (85) & $45^{\circ} 00^{\prime} 10^{\prime \prime} \mathrm{W} / 21^{\circ} 14^{\prime} 30^{\prime \prime} \mathrm{S}$ & Landa \& Mourgués-Schurter 2000b \\
\hline & Represa Pomar - UFLA (86) & $45^{\circ} 00^{\prime} 10^{\prime \prime} \mathrm{W} / 21^{\circ} 14^{\prime} 30^{\prime \prime} \mathrm{S}$ & Landa \& Mourgués-Schurter 1999 \\
\hline Jequitinhonha & Rio Jequitinhonha - Diamantina (87) & $43^{\circ} 30^{\prime} 52^{\prime \prime} \mathrm{W} / 18^{\circ} 24^{\prime} 20^{\prime \prime} \mathrm{S}$ & Landa 1997 \\
\hline Pardo & Reservatório Machado Mineiro (88) & $41^{\circ} 12^{\prime} \mathrm{W} / 15^{\circ} 31^{\prime} \mathrm{S}$ & Eskinazi-Sant'Anna et al. 2005 \\
\hline
\end{tabular}

a bacia do rio Pardo, com 8 espécies, e a do rio Jequitinhonha, com apenas 3 espécies (Figura 2).

Com base nos dados apresentados na Tabela 1 é possível verificar o número de corpos de água onde cada espécie foi registrada e com base nos dados das coordenadas geográficas e localização dos corpos de água nas bacias hidrográficas (Tabela 2) avaliar a amplitude da distribuição geográfica das diferentes espécies. A análise destes dados revela padrões bastante distintos para a distribuição espacial das 
espécies no estado de Minas Gerais. Na Figura 3 são exemplificadas algumas espécies de ampla distribuição.

Ceriodaphnia cornuta da família Daphnidae foi a espécie com a mais ampla distribuição, ocorrendo em 47 dos 88 corpos de água amostrados e com ocorrência em seis bacias hidrográficas. A segunda maior amplitude de distribuição correspondeu a Moina minuta, espécie da família Moinidae, com ocorrência em 36 dos 88 corpos de água amostrados e ocorrência em cinco bacias hidrográficas. As espécies Diaphanosoma birgei (Sididae) e Alona guttata (Chydoridae) também tiveram ampla distribuição. $D$. birgei ocorreu em 30 corpos de água e em quatro das seis bacias hidrográficas enquanto A. guttata (Chydoridae) ocorreu em 19 corpos de água e em quatro das seis bacias hidrográficas.

\section{Discussão}

Atualmente o conhecimento sobre a diversidade dos invertebrados aquáticos tem avançado rapidamente, devido às pesquisas diretamente direcionadas para o conhecimento da diversidade (Moretto 2001,
Miranda \& Pinto-Coelho no prelo, Pinto-Coelho et al. 2005a,b, Rocha 2006, Santos 1980, Santos et al. 2009, Santos-Wisniewski 1998) e também aos inventários relativos às avaliações de impacto ambiental (EIA-RIMA) para o licenciamento dos empreendimentos hidroelétricos ou em avaliações de viabilidade de implantações de parques aquícolas (Pinto-Coelho et al. 1997c, Pinto-Coelho 2001).

Os resultados obtidos no presente inventário revelaram que as águas doces do Estado de Minas Gerais contêm uma elevada riqueza de espécies de Cladocera quando comparada à riqueza de espécies conhecida para outros estados e para o Brasil. Esta representa cerca de $60 \%$ do total de espécies de Cladocera registradas no Brasil e é similar ao número de espécies registradas no estado de São Paulo (96 espécies) (Rocha et al. 2010).

Estes números revelam um considerável aumento no conhecimento nos últimos 15 anos. Em relação ao número de espécies de Cladocera nas águas continentais brasileiras Rocha et al. (1995) reportaram a ocorrência de 84 espécies, e Elmoor-Loureiro (1997) 158 espécies.

Para o estado de São Paulo Rocha \& Guntzel (1999) reportaram a ocorrência de 84 espécies e Rocha et al. (2010) recentemente

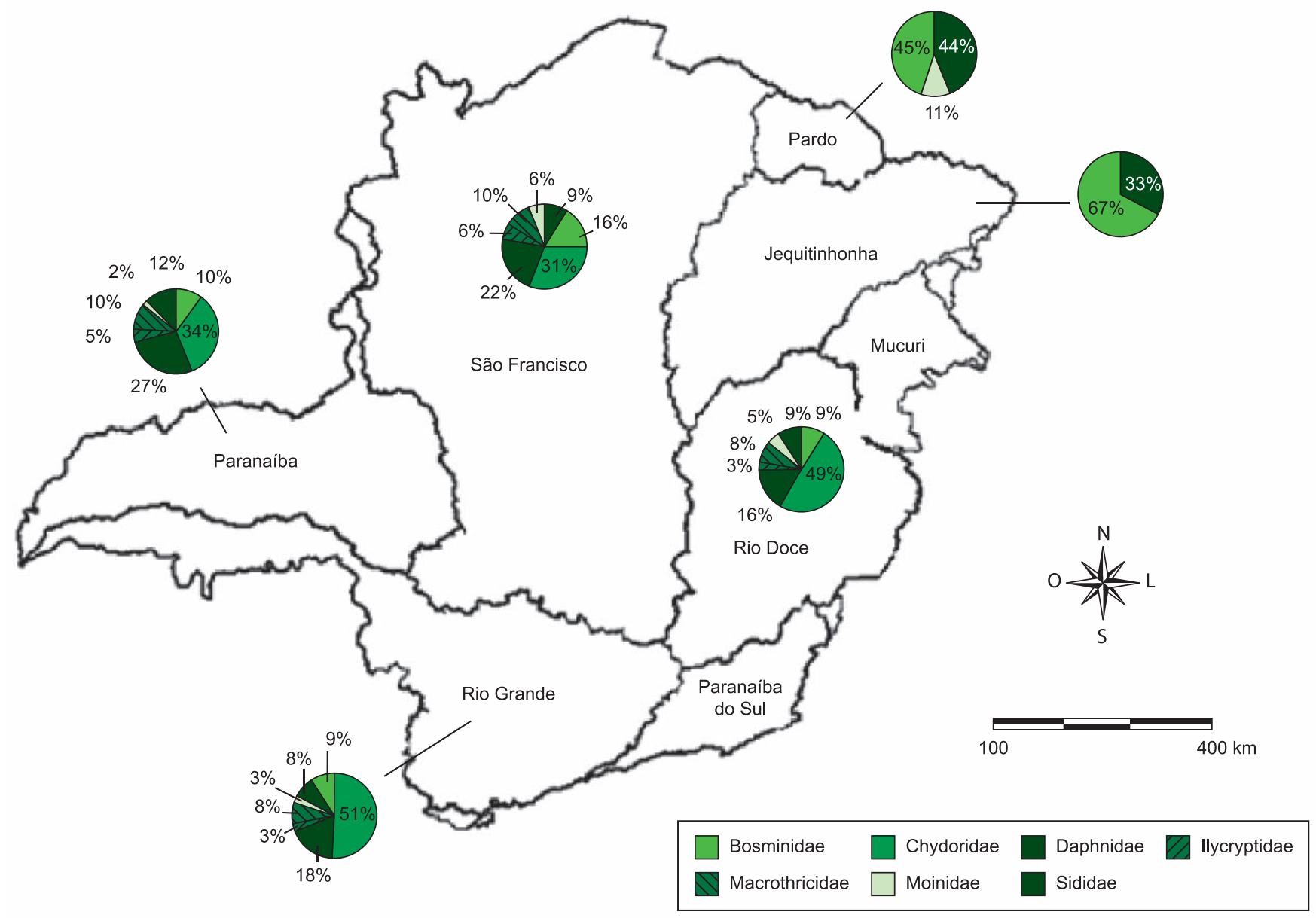

Figura 1. Percentual de contribuição de cada família de Cladocera por bacia hidrográfica, no estado de Minas Gerais.

Figure 1. Contribution percentage of each family of Cladocera by hydrographic basin, in Minas Gerais State. 
apresentaram uma check-list atualizada com 96 espécies já registradas, representando um aumento de $15 \%$ na riqueza de espécies de Cladocera para este estado, o que consideraram como um resultado positivo diretamente relacionado ao Programa Biota/Fapesp. Assim, nos últimos 10 anos o número de espécies reportadas tem aumentado, indicando um avanço significativo do conhecimento da diversidade destes invertebrados aquáticos.

Especificamente para o estado de Minas gerais, um primeiro inventário realizado por Maia-Barbosa et al. (2009) resultou no registro de 68 espécies de Cladocera em cinco bacias hidrográficas. Portanto, o presente inventário com o total de 94 espécies representa um aumento em torno de $39 \%$ das espécies anteriormente registradas. Contudo é amplamente reconhecido que este conhecimento é ainda incipiente. Os resultados do presente estudo evidenciam que no estado de Minas Gerais o número de espécies tem uma relação direta com o número de estudos realizados ou de corpos de água amostrados em cada bacia hidrográfica. Em algumas bacias foram feitos poucos ou nenhum estudo realizado até o presente, como no caso da bacia do rio Jequitinhonha, com apenas um estudo preliminar (Landa 1997) e as bacias dos rios Mucuri e Paraíba do Sul para as quais não foram encontrados dados sobre a diversidade da comunidade zooplanctônica.

Assim, há expectativas de que a riqueza de Cladocera no estado de Minas Gerais seja mais elevada e que aumentará significativamente nos próximos anos com os avanços previstos no âmbito do Programa BIOTA/MINAS.

Outro fato importante refere-se às espécies atualmente consideradas cosmopolitas ou aquelas com distribuição registrada nos dois hemisférios. Diversos autores têm recentemente mostrado que muitas destas espécies eram na realidade complexos de espécies morfologicamente similares, mas que à medida que foram sendo estudadas por especialistas e com técnicas mais avançadas resultaram na descrição de novas espécies, particularmente no Brasil. Um exemplo é o caso do gênero Ilyocryptus para o qual apenas três espécies eram conhecidas: Ilyocryptus spinifer Herrick, 1882, Ilyocryptus verrucosus Daday, 1905 e Ilyocryptus sordidus (Liévin 1848) (Sousa et al. 2010). Recentes revisões levaram ao esclarecimento de inúmeras confusões

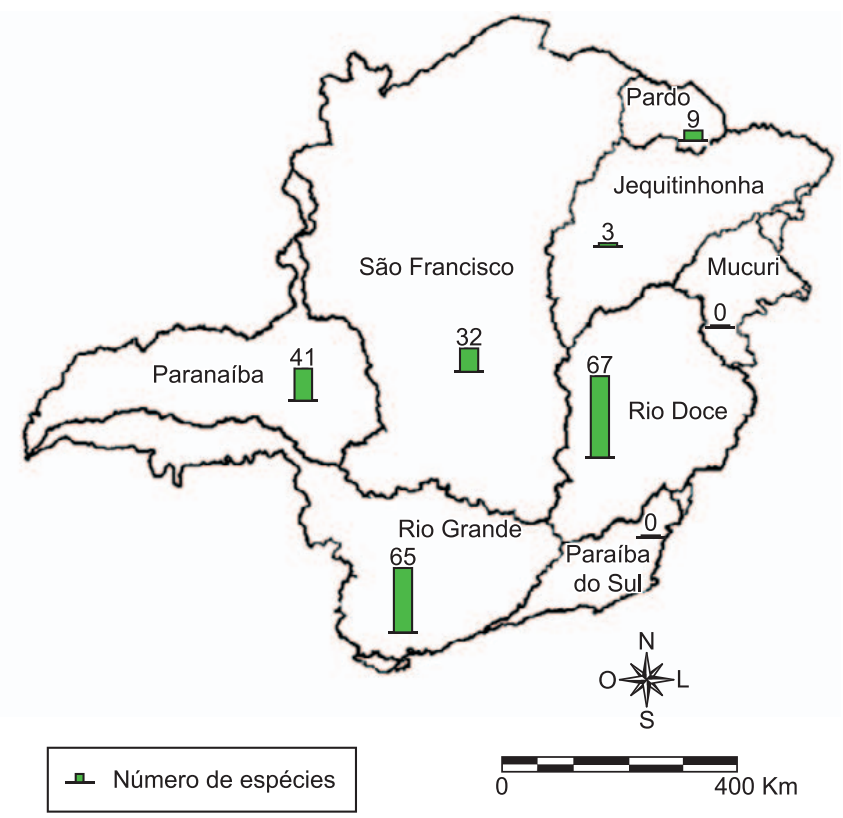

Figura 2. Número total de espécies de Cladocera já registrados nas principais bacias hidrográficas do estado de Minas Gerais.

Figure 2. Species total number of Cladocera ever recorded in the main hydrographic basin of the state of Minas Gerais. taxonômicas como a sinonímia de I. verrucosus com I. spinifer (Kotov \& Dumont 2000) e trabalhos recentes realizados com o auxílio de especialistas (Kotov et al. 2002, Kotov \& Stifter 2006, Kotov \& Elmoor-Loureiro 2008) permitiram a descrição de três novas espécies, elevando para cinco o número de espécies deste gênero no Brasil: Ilyocryptus cuneatus brasiliensis Kotov \& Elmoor-Loureiro 2008, Ilyocryptus paranaensis paranaensis Paggi 1989, Ilyocryptus sarsi Stingelin 1913, Ilyocryptus silvaeducensis paraensis Kotov \& Elmoor- Loureiro 2008 e Ilyocryptus spinifer.

Espécies de outras famílias, como os Daphnidae Daphnia ambigua Scourfield, 1947, Simocephalus serrulatus Koch, 1841; Scapholeberis armata Herrick, 1882 e Acroperus harpae são provavelmente complexos de espécies (Taylor et al. 1998, Elías-Gutiérrez \& Varella 2009, Sinev \& Elmoor-Loureiro 2010), e futuras revisões taxonômicas acopladas a estudos ecofisiológicos e genético-moleculares poderão revelar a diversidade críptica e os prováveis endemismos existentes.

Neste estudo algumas espécies tiveram seus nomes atualizados devido às revisões taxonômicas recentes. Assim, antes da revisão feita por Korinek (1981), a espécie Diaphanosoma birgei, uma das espécies com ampla distribuição no estado de Minas Gerais, havia sido confundida com Diaphanosoma brachyurum, uma espécie muito próxima (Elmoor-Loureiro 1990).

Diversas alterações foram também feitas para táxons da família Chydoridae. Alguns gêneros desta família foram recentemente revisados, como Alona, Chydorus, Camptocercus. Na recente revisão feita por van Damme \& Dumont (2008) a espécie Alona rectangula foi classificada em novo gênero, Coronatella, correspondendo na presente check list à espécie Coronatella rectangula. Alonella fitzpatricki Chien, 1970, anteriormente considerada um sinônimo júnior de Alona eximia, foi separada do gênero Alona e atribuída ao gênero Nicsmirnovius (van Damme et al. 2003).

O complexo de espécies Alona affinis encontra-se em estudo podendo vir a constituir um subgênero ou um gênero diferente de Alona (van Damme et al. 2010). Alguns espécimes do Brasil, oriundos da coleção de G.O. Sars foram identificados como uma nova espécie, A. ossiani (Sinev 1998). Desta forma os atuais registros de A. affinis no estado de Minas Gerais também necessitam confirmação.

A ocorrência de Alona quadrangularis (O.F. Müller, 1776) no Brasil (Pará, Mato Grosso do sul, Minas Gerais, Rio de Janeiro, São Paulo e Paraná), de acordo com Elmoor-Loureiro (2010), trata-se possivelmente de Alona yara. O mesmo ocorre para Graptoleberis occidentalis que de acordo com a mesma autora corresponde a G. testudinaria e para Acroperus harpae que provavelmente corresponde a Acroperus tupinamba. Em relação a Camptocercus Stingelin, 1913, Smirnov (1998) fez uma revisão deste gênero e considerou $C$. dadayi como sinônimo júnior de $C$. australis. Esta é uma questão ainda em aberto, que necessita de mais estudos (Elmoor-Loureiro 2010). Kotov (2009), na revisão para o gênero Leydigia, considera que os registros de Leydigia cilita no Brasil devem atribuídos a $L$. striata. Ainda, segundo este autor, a espécie Leydigia propinqua, que está listada neste estudo, tem distribuição restrita à África e os registros desta espécie na América do Sul, devem possivelmente ser erros de identificação. A ocorrência de L. ipojucae em Minas gerais, também necessita de confirmação.

Alona costata é outra espécie que deverá ser revista, pois de acordo com Sinev (2001) esta espécie corresponde a um complexo de espécies e nem A. costata nem A. rustica ocorreriam na América do Sul, de modo que os registros destas espécies no Brasil deveriam ser atribuídos a $A$. iheringula.

Sinev (2001) considera que os representantes sul-americanos do complexo Alona pulchella-cambouei, ou seja, os registros da ocorrência de A. cambouei e de A. pulchella no Brasil deveriam ser 


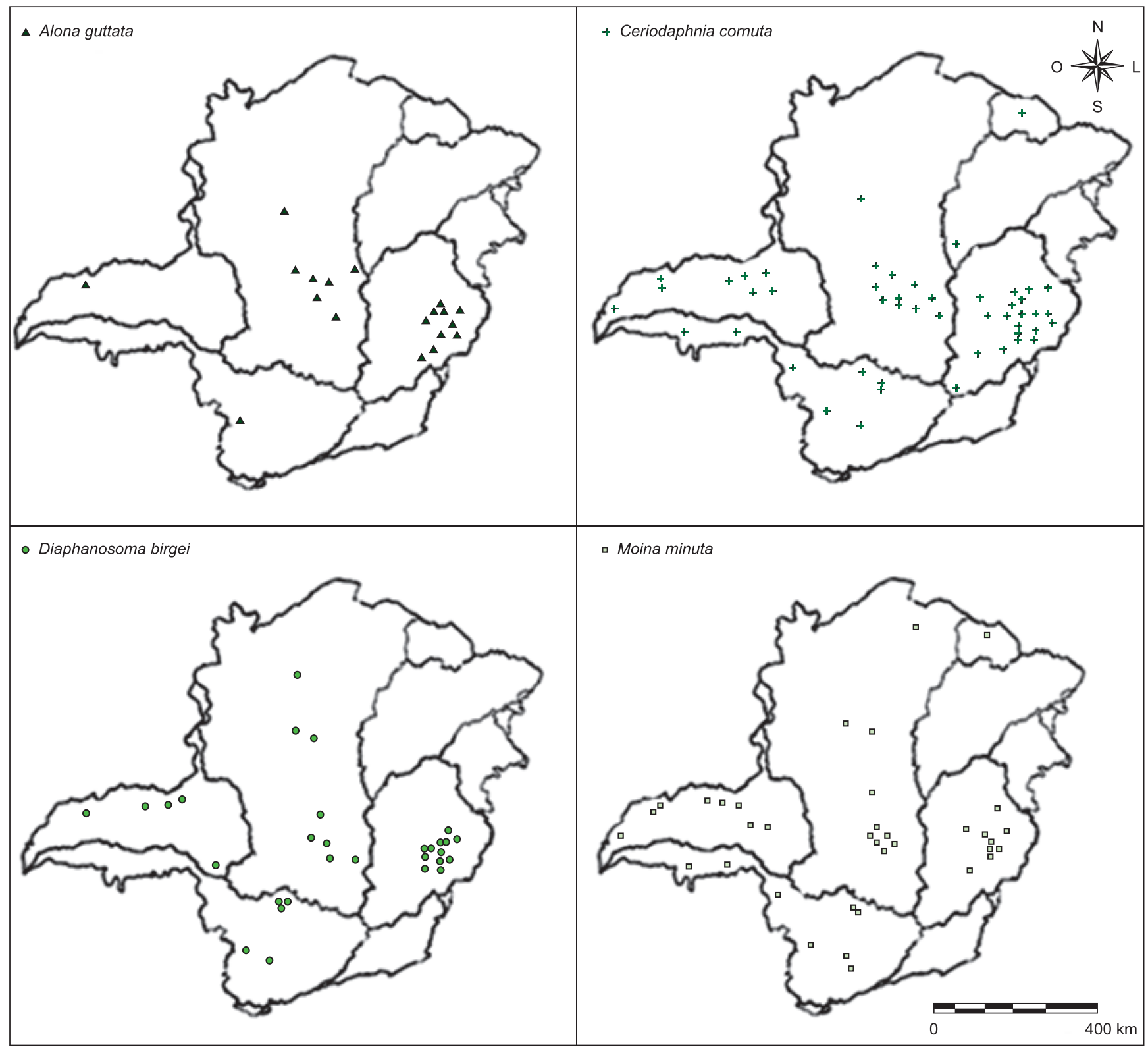

Figura 3. Mapa da distribuição das espécies mais abundantes para as principais famílias de Cladocera: Alona guttata (Chydoridae), Ceriodaphnia cornuta (Daphnidae), Diaphanosoma birgei (Sididae) e Moina minuta (Moinidae), no estado de Minas Gerais.

Figure 3. Map of distribution of most abundant species for the main families of Cladocera: Alona guttata (Chydoridae), Ceriodaphnia cornuta (Daphnidae), Diaphanosoma birgei (Sididae) and Moina minuta (Moinidae), in Minas Gerais state.

atribuídos a A. glabra. Esta também é a posição de van Damme et al. (2010).

Na presente check-list para os Cladocera do estado de Minas Gerais são relacionadas as espécies Alonella clathratula e Alonella excisa. A. clathratula era anteriormente considerada uma variedade de A. excisa, mas de acordo com Smirnov (1996) os registros desta última espécie no Brasil deveriam ser atribuídos a A. clathratula.

Os registros da ocorrência de Euryalona brasiliensis devem ser reavaliados, pois existem muitas semelhanças entre esta espécie e os exemplares brasileiros de Kurzia polyspina. Rajapaksa \& Fernando (1987) acreditam que esta espécie possa ser transferida para o gênero Kurzia. Segundo Elmoor-Loureiro (2010) a ocorrência de Kurzia latissima também precisa ser investigada, pois é provável que os registros desta espécie no Brasil correspondam a K. polyspina.
Na revisão realizada por Frey (1993) as espécies Pleuroxus similis e Pleuroxus cf. scopulifer são consideradas pertencentes ao subgênero Picripleuroxus, o qual a partir de Smirnov (1996) adquire o status de gênero. Amostragens nas localidades onde existem dúvidas quanto a taxonomia destas espécies, face às recentes revisões ou novas descrições, bem como estudos em novas localidades são urgentemente necessários para clarificar todas estas questões pendentes.

Adicionalmente, alguns registros de espécies são incertos por se tratarem de espécies com nomes atualmente inválidos ou que especialistas consideram não ocorrerem no Hemisfério Sul, como são os casos de Alona quadrata e Alonella nana registradas nos lagos do Vale do rio Doce (Moretto 2001, Miranda 2005) e de Bosmina coregoni, Daphnia dubia no rio Doce e Daphnia galeata na lagoa Amarela e no reservatório de Emborcação 
(Eskinazi-Sant'Anna et al. 2005). Estas espécies foram incluídas na presente check-list, mas, necessitam de revisão ou confirmação.

Considerando-se os aspectos acima apresentados é provável que o número de espécies com ocorrência no estado de Minas Gerais até a presente data seja ligeiramente inferior ao da lista aqui apresentada.

Adicionalmente, tem se enfatizado cada vez mais que a taxonomia de Cladocera necessita ser revista à luz de estudos mais aprofundados envolvendo não apenas o estudo morfológico, mas incluindo técnicas mais avançadas como a microscopia eletrônica, os estudos genético-moleculares e os estudos ecológicos para a maioria das espécies.

Neste estudo foi possível detectar também que as maiores riquezas de espécies em algumas bacias como a bacia do Rio Doce e a do Rio Grande resultam provavelmente do maior número de estudos nelas realizados. Conclui-se que há necessidade urgente de se ampliar a cobertura geográfica para amostragem dos corpos de água em outras bacias, particularmente aquelas localizadas na região norte e nordeste do estado de Minas Gerais.

\section{Agradecimentos}

Às agências FAPEMIG, FAPESP, CNPq e CAPES pelos auxílios à pesquisa e bolsas concedidas, no âmbito dos quais os estudos da diversidade do zooplâncton no Brasil estão sendo desenvolvidos; e à Dra. Lourdes M. A. Elmoor-Loureiro pela confirmação de algumas identificações e disponibilização de trabalhos.

\section{Referências Bibliográficas}

ALEMEIDA, C.G., PINESE, O.P. \& PINESE, J.F. 2007. Variação sazonal da comunidade de Cladocera no lago do Parque do Sabiá, (Uberlândia - MG). In Anais do VIII Congresso de Ecologia do Brasil. Sociedade de Ecologia do Brasil, Caxambu.

BEZERRA-NETO, J.F. 2001. A influência da larva de Chaoborus (Insecta: Diptera) na distribuição espacial da comunidade zooplanctônica na lagoa do Nado, Belo Horizonte - MG. Dissertação de Mestrado, Universidade Federal de Minas Gerais, Belo Horizonte.

BEZERRA-NETO, J.F. \& PINTO-COELHO, R.M. 2002. A influência da larva de Chaoborus (Insecta: Diptera) na distribuição espacial da comunidade zooplanctônica da Lagoa do Nado, BH - MG. Acta Sci., Biol. Sci. 24(2):337-344.

BEZERRA-NETO, J.F. \& PINTO-COELHO, R.M. 2003. Avaliação da eficiência de dois amostradores na estimativa da abundância e biomassa de organismos planctônicos. Bios, 11(11):19-27.

BEZERRA-NETO, J.F. \& PINTO-COELHO, R.M. 2008. Morphometric study of Lake Dom Helvécio, Parque Estadual do Rio Doce (PERD), Minas Gerais, Brazil: a re-evaluation. Acta Limnol. Bras. 20(2):161-167.

BRANDÃO, E.C.L., BRAZ, S.A., BRITO, S.L. MENENDEZ, R.M. \& MAIA-BARBOSA, P.M. 2002. Flutuação mensal do zooplâncton dos lagos Carioca, Dom Helvécio e Gambazinho (Parque Estadual do Rio Doce). In Dinâmica biológica e a conservação da biodiversidade da Mata Atlântica do médio Rio Doce, MG (PELD-UFMG) (F.A.R Barbosa, coord.). http://www.icb.ufmg.br/peld/ufmg/peld/relatorio2002. pdf (último acesso em 16/11/2010)

BRITO, S.L. 2010. Caracterização limnológica e produtividade secundária das principais espécies de microcrustáceos em dois braços dos reservatórios de Três Marias e Furnas, Minas Gerais, Brasil. Tese de doutorado, Universidade Federal de Minas Gerais, Belo Horizonte. http://ecologia. icb.ufmg.br/ rpcoelho/RioDoce/website/pdf/bach_1.pdf (último acesso em 14/11/2010).

BRITO, S.L, VALADARES, C.F., MENENDEZ, R.M. \& MAIA-BARBOSA, P.M. 2002. Estrutura da Comunidade Zooplanctônica em Três lagoas do entorno do Parque Estadual do Rio Doce (MG). In Dinâmica biológica e a conservação da biodiversidade da Mata Attântica do médio Rio Doce, MG (PELD-UFMG) (F.A.R Barbosa, coord.). http://www.icb.ufmg.br/ peld/ufmg/peld/relatorio2002.pdf (último acesso em 16/11/2010).
CARDOSO, L.S., RAMOS, J.D. \& MELO, H.O.O. 2008. Composição, densidade e abundância das populações de Cladocera, Copepoda e rotifera de Áreas de Proteção Permanente do rio Uberabinha. Em Extensão 7(2):95-106.

CASTILHO, M.C.A., SANTOS-WISNIEWSKI, M.J., VITI, T. 2009. Composição da comunidade zooplanctônica na entrada dos rios Verde e Sapucaí no reservatório da UHE de Furnas-MG. In Anais do XII Congresso Brasileiro de Limnologia. Sociedade Brasileira de Limnologia, Gramado.

CORGOSINHO, P.H.C. \& PINTO-COELHO, R.M. 2006. Zooplankton biomass, abundance and allometric patterns along an eutrophic gradient at Furnas Reservoir (Minas Gerais, Brazil). Acta Limnol. Bras. 18(2):213-224.

COSTA, A.R.G. 1998. Migração vertical diurna e teores de lipídeos do zooplâncton do reservatório da Pampulha (B.H./M.G.) e do reservatório de Furnas (Passos/M.G.). Dissertação de mestrado, Universidade Federal de Minas Gerais, Belo Horizonte.

DABÉS, M.B.G.S. 1995. Composição e descrição do zooplâncton de 5 (cinco) lagoas marginais do Rio São Francisco, Pirapora, Três Marias, Minas Gerais, Brasil. Rev. Bras. Biol. 55(4):831-845.

DEL ÁGUILA, L.M.R. 2001. Gradiente trófico no rio Sapucaí (Reservatório de Furnas-MG): relação com a distribuição do zooplâncton e os usos do solo. Dissertação de Mestrado, Universidade Federal de Minas Gerais, Belo Horizonte.

DUMONT, H. \& TUNDISI, J.G. 1997. Preliminary data on the paleolimnology of four lakes. In Limnological studies on the rio Doce Valley Lakes, Brazil (J.G. Tundisi \& Y. Saijo eds). Brazilian Academy of Sciences, University of São Paulo, São Carlos, p.457-474.

ELÍAS-GUTIÉRREZ, M. \& VARELA, C. 2009. An annotated checklist of the Cladocera of Cuba. Crustaceana 82(11):1353-1364. http://dx.doi.or $\mathrm{g} / 10.1163 / 001121609$ X12487811051426

ELMOOR-LOUREIRO, L.M.A. 1997. Manual de Invertebrados Límnicos do Brasil. Funiversa, Brasília.

ELMOOR-LOUREIRO, L.M.A. 1990. Diaphanosoma birgei e Diaphanosoma brachyurum: possível necessidade de revisão das identificações no Brasil. Acta Limnol. Bras. 3(2):757-767.

ELMOOR-LOUREIRO, L.M.A. 2010. Cladóceros do Brasil: Famílias Chydoridae e Eurycercidae. http://cladocera.wordpress.com/ (último acesso em 24/11/2010).

ESKINAZI-SANT'ANNA, E.M., MAIA-BARBOSA, P.M., BRITO, S. \& RIETZLER, A.C., 2005. Zooplankton biodiversity of Minas Gerais State: preliminary synthesis of present knowledge. Acta Limnol. Bras. 17(2): 199-218.

FERRARI, C.R. 2007. Distribuição espacial e temporal da comunidade zooplanctônica no reservatório de Furnas, com uma comparação entre os rios Grande e Sapucaí. Trabalho de Conclusão de Curso, Universidade Federal de Alfenas, Alfenas.

FERRAZ, H.D.A., LANDA, G.G. \& PAPROCKI, H. 2009. Zooplankton of an urban stretch, Itapecerica river, Divinópolis, Minas Gerais, Brazil. Check List 5(4):890-894.

FRANCO, T.V., NEGREIROS, N.F., FERRARI, C.R. \& SANTOS-WISNIEWSKI, M.J. 2005. Levantamento de espécies da comunidade zooplanctônica do reservatório de Furnas (Rio São Thomé), próximo à cidade de Alfenas-MG. In VII Congresso de Ecologia do Brasil. Sociedade de Ecologia do Brasil, Caxambu.

FREIRE, B. \& PINTO-COELHO, R.M. 1986. Composição e distribuição horizontal do zooplâcnton no reservatório de Vargem das Flores, Betim/Contagem, Minas Gerais. Ciênc. Cult. 38(5):919-928.

FREITAS, L.D. \& ESKINAZI-SANT'ANNA, E.M. 2009. Variações em curto prazo da comunidade zooplanctônica em lagoas de altitude do Parque Estadual do Itacolomi, Ouro Preto. In Anais do IX Congresso de Ecologia do Brasil. Sociedade de Ecologia do Brasil, São Lourenço.

FREY, D.G. 1993. Species of Pleuroxus (Anomopoda, Chydoridae) from the subantartic islands and southernmost South America: a partial unreavelling of Pleuroxus aduncus problem. Hydrobiologia 262(3):145-188. http:// dx.doi.org/10.1007/BF00010882 
GOMES E SOUZA, M.B. \& VON SPERLING, E. 2005. Uso do zooplâncton como indicador de qualidade da água - estudo de caso da bacia do rio Araguari - MG. In XXIII Congresso Brasileiro de Engenharia Sanitária e Ambiental. Associação Brasileira de Engenharia Sanitária e Ambiental, Campo Grande, Mato Grosso do Sul. http://www.bvsde.paho.org/bvsacd/ abes23/IV-001.pdf (último acesso em 21/07/2010).

HUDEC, I. 2000. Subgeneric differentiation within Kurzia (Crustacea; Anomopoda; Chydoridae) and a new species from Central America. Hydrobiologia 421(1):165-178. http://dx.doi. org/10.1023/A:1003919712255

JARAMILLO-LONDOÑO, J.C. \& PINTO-COELHO, R.M. 2010. Interaction between Hexarthra intermedia and Bosmina longirostris: A case of opportunistic nutrition or interference competition? J. Plankton Res. 32(6):961-966.

ISMAEL, D., VALENTE, C.V., MATSUMURA-TUNDISI, T. \& ROCHA, O. 1999. Invertebrados de Água Doce. In Biodiversidade do Estado de São Paulo, Brasil: síntese do conhecimento ao final do século XX (C.A. Joly \& C.E.M. Bicudo, orgs.). FAPESP, São Paulo.

KORINEK, V. 1981. Diaphanosoma birgei n. sp (Crustacea, Cladocera). A new species from America an its

widely distributed species Diaphanosoma birgei lacustris n. spp. Can. J. Zool. 59(6):1115-1121. http://dx.doi.org/10.1139/z81-155

KOTOV, A.A. 2003. Notes on Aloninae Dybowski \& Grochowski, 1894 emend. Frey, 1967 (Cladocera: Anomopoda: Chydoridae). 1. Translocation of Alona incredibilis Smirnov, 1984 to the genus Nicsmirnovius Chiambeng \& Dumont, 1999. Arthrop. Selecta 12(34):167-170.

KOTOV, A.A. 2009. A revision of Leydigia Kurz, 1875 (Anomopoda, Cladocera, Branchiopoda), and subgeneric differentiation within the genus. Zootaxa 2082:1-84.

KOTOV, A.A. \& DUMONT, H.J. 2000. Analysis of the spinifer-species group (Anomopoda, Branchiopoda), with description of a new species. Hydrobiologia 428(1-3):85-113. http://dx.doi. org/10.1023/A:1003977208875

KOTOV, A.A. \& SINEV, A.Y. 2004. Notes on Aloninae Dybowski \& Grochowski, 1894 emend. Frey, 1967 (Cladocera: Anomopoda: Chydoridae): 3. Alona iheringula nom. nov. instead of A. iheringi Sars, 1901, with comments on this taxon. Arthrop. Selecta 13(3):95-98.

KOTOV, A.A. \& STIFTER, P. 2006. Family Ilyocryptidae (Branchiopoda: Cladocera: Anomopoda). Backuys Publishers, Leiden.

KOTOV, A.A. \& ELMOOR-LOUREIRO, L.M.A. 2008. Revision of Ilyocryptus Sars, 1862 (Cladocera: Ilyocryptidae) of Brazil whit description of two new subspecies. Zootaxa 1962: 49-64.

KOTOV, A.A., DUMONT, H.J. \& VAN DAMME, K. 2002. Redescription of Ilyocryptus sarsi Stingelin, 1913. Hydrobiologia 472(1-3):207-222. http://dx.doi.org/10.1023/A:1016373226707

LANDA, G.G. 1997. Contribuição ao estudo da comunidade zooplanctônica em uma área sob influência de mineração na bacia do rio Jequitinhonha - MG. Bios 5(5):69-80.

LANDA, G.G. \& MOURGUÉS-SCHURTER, L.R. 1999. Composição e abundância do zooplâncton em um sistema artificial raso (Represa Pomar) no campus da Universidade Federal de Lavras-Minas Gerais. Bios, 7(7):21-31

LANDA, G.G. \& MOURGUÉS-SCHURTER, L.R. 2000a. Composição e abundância do zooplâncton de duas represas do campus da Universidade Federal de Lavras-MG. Acta Limnol. Bras. 12(2):29-43.

LANDA, G.G.; MOURGUÉS-SCHURTER, L.R. 2000b. Caracterização da comunidade zooplanctônica de um sistema artificial (represa Zootecnia) no campus da Universidade Federal de Lavras - MG. Acta Limnol. Bras. 12(2):63-68.

LANDA, G.G. \& TÔRRES, G.E. 2004. Distribuição vertical do zooplâncton e avaliação de parâmetros físico-químicos da Lagoa Olhos d'água - Lagoa Santa/MG. Sinapse Amb. 1(4):23-35.
LEONE, I.C. 2009. Distribuição espacial de Ceratium furcoides (Dinoflagellata: Ceratiaceae) no reservatório da UHE de Furnas e sua influência sobre a comunidade zooplanctônica. Trabalho de conclusão de curso, Universidade Federal de Alfenas, Alfenas.

LÓPEZ, C.M., VONO, V. \& MAIA-BARBOSA, P.M. 1996. Avaliaçäo da comunidade zooplanctônica na regiäo litorânea de dois lagos naturais no Médio Rio Doce. Arq. Bras. Med. Vet. Zootec. 48(supl. 1):141-149.

MACEDO, C.F. \& PINTO-COELHO, R.M. 1997. A influência do ritmo circadiano nas taxas de respiração do zooplâncton da Lagoa da Pampulha. Acta Limnol. Bras. 9(1):125-137.

MACEDO, C.F. \& PINTO-COELHO, R.M. 2000a. Diel variations in respiration, excretion rates and nutritional status of zooplankton from the Pampulha Reservoir, Belo Horizonte. J. Exp.Zool. 286(7):671-682. http:// dx.doi.org/10.1002/(SICI)1097-010X(20000601)286:7\%3C671::AIDJEZ1\%3E3.0.CO;2-E

MACEDO, C.F. \& PINTO-COELHO, R.M. 2000b. Efeitos das algas Ankistrodemus gracilis e Scenedesmus quadricauda no crescimento e no índice lipídico de Daphnia laevis e Moina micrura. Acta Sci., Biol. Sci. 22(2):397-401

MACEDO, C.F. \& PINTO-COELHO, R.M. 2000c. Taxas de filtração de Daphnia laevis e Moina micrura em relação às clorofíceas Scenedesmus quadricauda e Ankistrodesmus gracilis. Acta Limn. Bras. 12(1):1-10.

MAIA-BARBOSA, P.M., MENENDEZ, R.M., ESKINAZI-SANT'ANNA, E.M. \& PINTO, M.T.C. 2003. Zooplâncton de uma lagoa marginal do alto São Francisco. In Águas, peixes e pescadores do São Francisco das Minas Gerais (H.P. Godinho \& A.L. Godinho, orgs.). PUC Minas, Belo Horizonte, p.105-113.

MAIA-BARBOSA, P.M., PEIXOTO, R.S. \& GUIMARÃES, A.S. 2008. Zooplankton in littoral waters of a tropical lake: a revisited biodiversity. 2008. Braz. J. Biol. 68(Suppl. 4):1069-1078. http://dx.doi.org/10.1590/ S1519-69842008000500014

MAIA-BARBOSA, P.M., MACHADO, C.F., BARBOSA, F.A.R., FERREIRA, H.L.M., BRITO, S.L., JUNQUEIRA, M.V., CAMPOS, M.C.S., MEYER, S.T., JARDIM, B.F.M., GAMA, F.O., MOTA, H.R \& MENENDEZ, R.M. 2009. Diversidade de organismos aquáticos. In Biota Minas. Diagnóstico do Conhecimento sobre a Biodiversidade no estado de Minas Gerais (G.M. Drummond, C.S. Martins, M.B. Greco \& F. Vieira). Programa BIOTA MINAS, Fundação Biodiversitas, Belo Horizonte, p.81-121.

MATSUMURA-TUNDISI, T. 1997. Composition and vertical distribution of zooplankton in lake Dom Helvécio. In Limnological studies on the rio Doce Valley Lakes, Brazil. (J.G. Tundisi \& Y. Saijo, eds). Brazilian Academy of Sciences, University of São Paulo, São Carlos, p.265-274.

MATSUMURA-TUNDISI, T., TUNDISI, J.G. \& TAVARES, L.S. 1984. Diel migration and vertical distribution of Cladocera in lake D. Helvécio (Minas Gerais, Brazil). Hydrobiologia 113(1): 299-306. http://dx.doi. org/10.1007/BF00026616

MATSUMURA-TUNDISI, T., TUNDISI, J.G., ROCHA, O. \& CALIJURI, M.C. 1997. The Ecological significance of the metalimnion in Lakes of middle rio Doce Valley. In Limnological studies on the rio Doce Valley Lakes, Brazil. (J.G. Tundisi \& Y. Saijo, eds). Brazilian Academy of Sciences, University of São Paulo, São Carlos, p.373-390.

MENENDEZ, R.M. \& MAIA-BARBOSA, P.M. 2002. Comunidade zooplanctônica de sete ambientes lóticos da Bacia do Médio Rio Doce (MG). In Dinâmica biológica e a conservação da biodiversidade da Mata Atlântica do médio Rio Doce, MG (F.A.R. Barbosa, coord.). UFMG. http://www.icb.ufmg.br/peld/ufmg/peld/relatorio2002.pdf (último acesso em 16/11/2010).

MIRANDA, F. \& PINTO-COELHO, R.M. Diminuição da riqueza em espécies e da diversidade de Cladocera em lagos do Médio Rio Doce. Rev. Bras. Zoociências :no prelo.

MIRANDA, F.S. 2005. Depleção da riqueza zooplanctônica no Médio Rio Doce. Monografia de Bacharelado. Universidade Federal de Minas Gerais. http://ecologia.icb.ufmg.br/ rpcoelho/RioDoce/website/pdf/bach_3.pdf (último acesso em 18/11/2010). 
MORETTO, E.M. 2001. Diversidade zooplanctônica e variáveis limnológicas das regiões limnética e litorânea de cinco lagoas do Rio Doce e sua relação com o entorno. Dissertação de mestrado, Universidade de São Paulo, São Carlos.

PAES, T.A.S.V., SILVA, L.C., NEGREIROS, N.F., SANTOS, R.M. \& SANTOS-WISNIEWSKI, M.J. 2008. Riqueza e diversidade da comunidade zooplanctônica do reservatório da UHE de Furnas (rio Sapucaí) na região de Carmo do Rio Claro. In III Workshop da Pós-Graduação, X Mostra de Extensão e XIV Jornada Científica de Alfenas. UFAL, Alfenas.

PEIXOTO, R.S. \& OKANO, W.Y. 2004. Estrutura e flutuação temporal das populações zooplanctônicas (Copepoda e Cladocera) das represas da Reitoria e Quatro Pilastras, campus da Universidade Federal de Viçosa (UFV), MG. Bios 12(12):45-52.

PERET, A.C. 2007. Caracterização biológica da ictiofauna da represa Cachoeira Dourada. Universidade Federal de São Carlos, São Carlos, 42p. Relatório final.

PINESE, O.P. 2008. Ecologia de comunidades zooplanctônicas em lagoas associadas a veredas no município de Uberlândia, MG. Dissertação de mestrado, Universidade Federal de Uberlândia, Uberlândia.

PINTO-COELHO, R.M. 1998. Effects of eutrophication effects on seasonal patterns of mesozooplankton in a tropical reservoir: a four years study in Pampulha Lake, Brazil. Fresh. Biol. 40(1):159-174. http://dx.doi. org/10.1046/j.1365-2427.1998.00327.x

PINTO-COELHO, R.M. 2001. Melhoria da qualidade nutricional do zooplâncton usado como alimento em estações de piscicultura. FAPEMIG/CNPq, Relatório final. http://ecologia.icb.ufmg.br/ rpcoelho/ art_pdf/rf_cra1897.pdf (último acesso em 21/07/2010).

PINTO-COELHO, R.M. 2010. Biomonitoramento do reservatório da Pampulha. http://ecologia.icb.ufmg.br/ rpcoelho/atlas/cladoc.htm (último acesso em 01/12/2010)

PINTO-COELHO, R.M. \& CORGOSINHO, P.H. 1998. Alterações na estrutura do zooplâncton em resposta a um gradiente de trofia no reservatório de Furnas, Minas Gerais. In Anais do VIII Seminário Regional de Ecologia. Sociedade de Ecologia do Brasil, São Carlos, v.8, p.1173-1188.

PINTO-COELHO, R.M. \& GRECO, M.B. 1998. Teores de metais pesados em organismos zooplanctônicos e na macrófita Eichhornia crassipes na represa da Pampulha, Belo Horizonte, MG. Agua Rev. 12:64-69.

PINTO-COELHO, R.M. \& GRECO, M.B. 1999. The contribution of water hyacinth (Eichhornia crassipes) and zooplankton to the internal cycling of phosphorus in the eutrophic Pampulha Reservoir. Hydrobiologia 411(1):115-127. http://dx.doi.org/10.1023/A:1003845516746

PINTO-COELHO, R.M., SÁ JUNIOR, W.P. \& CORGOSINHO, P.H. 1997a. Variação nictimeral do status nutricional do zooplâncton em tanques de cultivo de plâncton. Rev. Unimar 19(2):521-535.

PINTO-COELHO, R.M., AMORIM, M.K. \& COSTA, A.R. 1997b. Temporal dynamics of lipids in the zooplankton of two tropical reservoirs with different trophic status. Ver. Int. Verh. Theor. Angew. Limnol. 26:584-587.

PINTO-COELHO, R.M., BEZERRA-NETO, J.F. \& MORAIS JUNIOR, C.A. 2005a. Effects of eutrophication on size and biomass of crustacean zooplankton in a tropical reservoir. Braz. J. Biol. 65(2):325-338. http:// dx.doi.org/10.1590/S1519-69842005000200017

PINTO-COELHO, R.M., BEZERRA-NETO, J.F., GIANI, A., MACEDO, C.F., FIGUEIREDO, C.C. \& CARVALHO, E.A. 2003. The collapse of a Daphnia laevis (Birge, 1878) population in Pampulha Reservoir, Brazil. Acta Limnol. Bras. 15(3):53-70.

PINTO-COELHO, R.M., BEZERRA-NETO, J.F., MIRANDA, F., MOTA, T.G., RESCK, R., SANTOS, A.M., MAIA-BARBOSA, P.M., MELLO, N.A.S.T., MARQUES, M.M., CAMPOS, M.O. \& BARBOSA, F.A.R. 2008. The inverted trophic cascade in tropical planktonic communities: impacts of exotic fish introduction in the Middle Rio Doce lake district, Minas Gerais, Brazil. Braz. J. Biol. 68 (Suppl. 4):1025-1037. http://dx.doi. org/10.1590/S1519-69842008000500010
PINTO-COELHO, R.M., GIANI, A., MORAIS JUNIOR, C.A., CARVALHO JUNIOR., E., BEZERRA-NETO, J.F. 2005b. The nutritional status of zooplankton in a tropical reservoir: effects of food quality and community structure. Braz. J. Biol. 65(2):313-324. http://dx.doi.org/10.1590/S151969842005000200016

PINTO-COELHO, R.M., MOURA, R.T. \& MOREIRA, A. $1997 \mathrm{c}$. Zooplankton and bacteria contribution to phosphorus and nitrogen internal cycling in a tropical and eutrophic reservoir: Pampulha Lake, Brazil. Int. Rev. Hydrobiol. 82(2):195-200.

PINTO-COELHO, R.M., NUNES, C., BARBEITOS, M., MORAIS, C. \& GUERRA, S.A. 1998. O impacto da refinaria Gabriel Passos - REGAP na estruturação da comunidade zooplanctônica no reservatório de Ibirité, Minas Gerais. Bios 6(6):11-19.

PINTO-COELHO, R.M., PINEL-ALLOUL, B., MÉTHOT, G. \& HAVENS, K. 2005c. Crustacean zooplankton in lakes and reservoirs of temperate and tropical regions: variations with trophic status. Can. J. Fish. Aquat. Sci. 61:348-361. http://dx.doi.org/10.1139/f04-178

RAJAPAKSA, R. \& FERNANDO, CH. 1987. A redescription of Euryalona orientalis (Daday 1898), with a consideration of the others species of the genus Euryalona (Cladocera, Chydoridae). Hydrobiologia 150(1):75-90. http://dx.doi.org/10.1007/BF00006611

RAMOS, J.D., MELLO, H.O.O. \& LIMA, L. 2008. Análise da composição, abundância e distribuição vertical das populações de Rotifera, Cladocera e Copepoda, no reservatório de Emborcação (Araguari - Mg). Em Extensão 7(2):80-94.

ROCHA, G.S. 2006. Variação anual da comunidade de Cladocera (Crustacea). Trabalho de Conclusão de Curso, Universidade Federal de Alfenas, Alfenas, Minas Gerais.

ROCHA, O. \& MATSUMURA-TUNDISI, T. 1997. Respiration rates of zooplankton community of epilimnion and metalimnion layers of lake Dom Helvécio during stratified and overturn periods. In Limnological studies on the rio Doce Valley Lakes, Brazil. (J.G. Tundisi \& Y. Saijo eds.). Brazilian Academy of Sciences, University of São Paulo, São Carlos, p.285-296.

ROCHA, O. \& GÜNTZEL, A.M. 1999. Crustáceos branquiópodos. In. Biodiversidade do estado de São Paulo, Brasil. Invertebrados de Água doce (D. Ismael, W.C. Valenti, T. Matsumura-Tundisi, \& O. Rocha, coords.). FAPESP, São Paulo, v. 4, p.107-120.

ROCHA, O., SENDACZ, S. \& MATSUMURA-TUNDISI, T. 1995. Composition, biomass and productivity of zooplankton in natural lakes and reservoirs in Brazil. In Limnology in Brazil. (J.G. Tundisi, C.E.M. Bicudo, T. Matsumura-Tundisi, orgs.). Academia Brasileira de Ciências, Rio de Janeiro, p.151-166.

ROCHA, O., ESPÍNDOLA, E.L.A., FENERICH-VERANI, N., VERANI, J.R. \& RIETZLER, A.C. 2005. (Orgs.) Espécies invasoras em águas doces : estudo de caso e propostas de manejo em São Carlos. EdUFSCar, São Carlos.

ROCHA,O., SANTOS-WISNIEWSKI, M.J. \& MATSUMURA-TUNDISI, T. 2010. Check list da Cladocera de água doce do estado de São Paulo. Biota Neotrop. 11(1a): http://www.biotaneotropica.org.br/v11n1a/en/ab stract?inventory+bn0271101a2011.

ROSA, P.G. 2008. Comunidade zooplanctônica de cinco reservatórios tropicais do sistema Furnas S.A. (Brasil): abundância e biomassa em carbono. Dissertação de Mestrado Universidade Federal de Juiz de Fora, Juiz de Fora.

SAMPAIO, E.V. 2002. Composição, abundância e diversidade das comunidades zooplanctônicas em reservatórios do sudeste do Brasil (Bacias dos rios Paranapanema e alto São Francisco). Tese de Doutorado, Universidade Federal de São Carlos, São Carlos.

SAMPAIO, E.V \& LÓPEZ, C.M. 2000. Zooplankton composition and some limnological aspects of an oxbow Lake of the Paraopeba River, São Francisco River Basin, Minas Gerais, Brazil. Braz. Arch. Biol. Technol. 43(3):285-293. http://dx.doi.org/10.1590/S151689132000000300007 
SANTEIRO, R.M. \& PINTO-COELHO, R.M. 2000. Efeitos do regime de fertilização na biomassa e qualidade nutricional de zooplâncton utilizado para a alimentação de alevinos na estação de hidrobiologia e piscicultura de Furnas, Minas Gerais. Acta Sci., Biol. Sci. 22(3):707-716.

SANTEIRO, R.M., PINTO-COELHO, R.M. \& SIPAÚBA, L.H. 2006. Diurnal variation of zooplankton biochemical composition and biomass in plankton production tanks. Acta Sci., Biol. Sci. 28(2):103-108.

SANTOS, L.C. 1980. Estudo das populações de Cladocera em cinco lagos naturais (Parque Florestal do Rio Doce - MG), que se encontram em diferentes estágios de evolução. Dissertação de Mestrado, Universidade Federal de São Carlos, São Carlos.

SANTOS, R.M. 2007. Diversidade e produção de Cladocera em quatro pontos do reservatório da UHE de Furnas-MG (rios Cabo Verde, Muzambão, Sapucaí e a junção dos três rios). Trabalho de Conclusão de Curso, Universidade Federal de Alfenas, Alfenas.

SANTOS, R.M., NEGREIROS, N.F. \& SANTOS-WISNIEWSKI, M.J. 2007. Diversidade e biomassa de Cladocera em quatro pontos do reservatório da UHE de Furnas (rios Cabo Verde, Muzambão, Sapucai e a junção dos três rios). In VIII Congresso de Ecologia do Brasil. Sociedade de Ecologia do Brasil, Caxambu.

SANTOS, R.M., NEGREIROS, N.F., ROCHA, O. \& SANTOS-WISNIEWSKI, M.J. 2008. Produção e biomassa de cladóceros e rotíferos no reservatório da UHE de Furnas - MG. In II Simpósio de Ecologia do Programa de Pós-Graduação em Ecologia e Recursos Naturais. Universidade Federal de São Carlos, São Carlos.

SANTOS, R.M., ROCHA, G., ROCHA, O. \& SANTOS-WISNIEWSKI, M.J. 2009. Influence of net cage fish cultures on the diversity of the zooplankton community in the Furnas hydroelectric reservoir, Areado, MG, Brazil. Aquac. Res. 40:753-761. http://dx.doi.org/10.1111/j.13652109.2008.02148.x

SANTOS-WISNIEWSKI, M.J. 1998. Distribuição espacial e produção secundária da comunidade zooplanctônica do Reservatório de Barra Bonita - SP. Tese de doutorado, Universidade Federal de São Carlos, São Carlos.

SANTOS-WISNIEWSKI, M.J., FERRARI, C.R., NEGREIROS, N.F. \& FRANCO, T.V. 2005. Levantamento de espécies da comunidade zooplanctônica do reservatório de Furnas (Rios Sapucaí e São Thomé), Alfenas-MG. In X Congresso Brasileiro de Limnologia, Ilhéus.

SILVA, L.C. 2011. Variação espacial e temporal da diversidade e biomassa da comunidade zooplanctônica em 35 pontos do reservatório da UHE de Furnas - MG. Dissertação de mestrado, Universidade Federal de São Carlos, São Carlos.

SILVA, L.C., FORMAGIO, P.S., FERREIRA, M.N.L. \& SANTOS-WISNIEWSKI, M.J. 2007. Levantamento das espécies da comunidade zooplanctônica do reservatório da UHE de Furnas - rios Machado e Sapucaí. In II Workshop da Pós-Graduação, IX Mostra de Extensão e XII Jornada da Iniciação Científica de Alfenas, Alfenas.

SILVA, E.S., CASTILHO, M.C.A., LUCCAS, P.O., WISNIEWSKI, C. \& SANTOS-WISNIEWSKI, M.J. 2009. Cladóceros litorâneos de dois pontos do Rio Cabo verde no Reservatório da UHE de Furnas. In Anais do XII Congresso Brasileiro de Limnologia. Sociedade Brasileira de Limnologia, Gramado.

SANTOS-WISNIEWSKI, M.J. 2009. Avaliação do aporte de nutrientes e de metais tóxicos e suas relações com a diversidade das comunidades planctônica e bentônica nos tributários da UHE Furnas - MG (Braço Sapucai). Projeto Plurianual Ciclo, Relatório Final.

SINEV, Y.A. 1998. Alona ossiani sp.n., a new species of the Alona affinis complex from Brazil, deriving from the collection of G.O. Sars (Anomopoda: Chydoridae). Arthrop. Selecta 7(2):103-110.

SINEV, A.Y. 2001. Redescription of Alona iheringi Sars, 1901 (Chydoridae, Anomopoda, Branchiopoda), a South American species related to A. rustica Scott, 1895. Hydrobiologia 464(1-3):113-119. http://dx.doi. org/10.1023/A:1013935100483
SINEV, A.Y. \& W. HOLLWEDEL. 2005. Translocation of Alona muelleri Richard, 1897 into the genus Karualona Dumont \& Silva-Briano, 2000 (Branchiopoda: Anomopoda: Chydoridae). Arthrop. Selecta 14(2):93-101.

SINEV, A.Y. \& ELMOOR-LOUREIRO, L.M.A. 2010. Three new species of chydorid cladocerans of subfamily Aloninae (Branchipoda: Anomopoda: Chydoridae) from Brazil. Zootaxa 2390:1-25.

SINEV, A. Y., van DAMME, K. \& KOTOV, A. A. 2005. Redescription of tropical-temperate cladocerans Alona diaphana King, 1853 and Alona davidi Richard, 1895 and translocation to Leberis Smirnov, 1989 (Branchiopoda:Anamopoda: Chydoridae). Arthrop. Selecta 14(3):183-205.

SIPAÚBA-TAVARES, L.H., SANTEIRO, R.M., PINTO-COELHO, R.M. \& BRAGA, F.M.S. 2009. Effect of fertilization in water quality and in zooplankton community in open plankton-culture ponds. Biosci. J. 25(3):172-180.

SOUSA, F.D.R., ELMOOR-LOUREIRO, L.M.A., MENDONÇA-GALVAO, L. \& FERNANDES JUNIOR, I.R. 2010. Crustacea, Cladocera, Anomopoda, Ilyocryptidae, Ilyocryptus sarsi Stingelin, 1913: a new record and geographic distribution in Brazil. Check List 6:330-331.

SOUZA, M.P. 2007. Organismos planctônicos de sistemas de lagoas de tratamento de esgotos sanitários como alimento natural na criação de tilápia do Nilo. Dissertação de mestrado, Universidade Federal de Viçosa, Viçosa.

SMIRNOV, N.N. 1996. Cladocera: The Chydorinae and Sayciinae (Chydoridae) of the world. SPB Academic Publishing, Amsterdam.

SMIRNOV, N.N. 1998. A revision of the genus Camptocercus (Anomopoda, Chydoridae, Aloninae). Hydrobiologia 386(1-3):63-83. http://dx.doi.org/10.1023/A:1003524414799

TAYLOR, D.J., FINSTON, T.L. \& HEBERT, P.D.N. 1998. Biogeography of a widespread freshwater crustacean: pseudocongruence and cryptic endemism in the North American Daphnia laevis complex. Evolution 52:1648-1670. http://dx.doi.org/10.2307/2411338

TUNDISI, J.G. 2003. Água no século XXI: enfrentando a escassez. RiMa, São Carlos.

TUNDISI, J.G., MATSUMURA-TUNDISI, T., FUKUARA, H., MITAMURA, O., ROCHA, O., GUILLÉN, S.M., HENRY, R., CALIJURI, M.C., IBAÑES, M.S.R., ESPÍNDOLA, E.L.G. \& GOVONI, S. 1997a. Limnology of fifteen lakes. In Limnological studies on the rio Doce Valley Lakes, Brazil. (J.G. Tundisi \& Y. Saijo, eds). Brazilian Academy of Sciences, University of São Paulo, São Carlos, p.409-440.

TUNDISI, J.G., MATSUMURA-TUNDISI, T., CALIJURI, M.C., HENRY, R., IBAÑES, M.S.R. \& NAKAMOTO, N. 1997b. Limnological survey of lake Anibal. In Limnological studies on the rio Doce Valley Lakes, Brazil (J.G. Tundisi \& Y. Saijo, eds). Brazilian Academy of Sciences, University of São Paulo, São Carlos, p.441-448.

VAN DAMME, K. \& DUMONT, H.J. 2008. Further division of Alona Baird, 1843: separation and position of Coronatella Dybowski \& Grochowski and Ovalona gen.n. (Crustacea: Cladocera). Zootaxa 1960:1-44.

VAN DAMME, K., KOTOV, A.A. \& DUMONT, H.J. 2010. A checklist of names in Alona Baird 1843 (Crustacea: Cladocera: Chydoridae) and their current status: an analysis of the taxonomy of a lump genus. Zootaxa, 2330:1-63.

VAN DAMME, K., CHIAMBENG, G., MAIPHAE, S. \& DUMONT, H.J. 2003. New species in the rheophilous genus Nicsmirnovius Chiambeng \& Dumont, 1999 (Branchiopoda: Anomopoda: Chydoridae) and reassignment of Alona eximia Kiser, 1948 and Alonella fitzpatricki Chien, 1970. Hydrobiologia 499(1-3):25-49. http://dx.doi. org/10.1023/A:1026391501312 